\title{
Comprehensive evaluation of genome-wide 5 -hydroxymethylcytosine profiling approaches in human DNA
}

Ksenia Skvortsova' ${ }^{1}$, Elena Zotenko' , Phuc-Loi Luu' ${ }^{1}$, Cathryn M. Gould ${ }^{1}$, Shalima S. Nair ${ }^{1,2}$, Susan J. Clark ${ }^{1,2^{*+}}$ (]) and Clare Stirzaker ${ }^{1,2^{*}+}$

\begin{abstract}
Background: The discovery that 5-methylcytosine $(5 \mathrm{mC})$ can be oxidized to 5-hydroxymethylcytosine (5hmC) by the ten-eleven translocation (TET) proteins has prompted wide interest in the potential role of $5 \mathrm{hmC}$ in reshaping the mammalian DNA methylation landscape. The gold-standard bisulphite conversion technologies to study DNA methylation do not distinguish between $5 \mathrm{mC}$ and $5 \mathrm{hmC}$. However, new approaches to mapping $5 \mathrm{hmC}$ genomewide have advanced rapidly, although it is unclear how the different methods compare in accurately calling $5 \mathrm{hmC}$. In this study, we provide a comparative analysis on brain DNA using three $5 \mathrm{hmC}$ genome-wide approaches, namely whole-genome bisulphite/oxidative bisulphite sequencing (WG Bis/OxBis-seq), Infinium HumanMethylation450 BeadChip arrays coupled with oxidative bisulphite (HM450K Bis/OxBis) and antibody-based immunoprecipitation and sequencing of hydroxymethylated DNA (hMeDIP-seq). We also perform loci-specific TET-assisted bisulphite sequencing (TAB-seq) for validation of candidate regions.
\end{abstract}

Results: We show that whole-genome single-base resolution approaches are advantaged in providing precise $5 \mathrm{hmC}$ values but require high sequencing depth to accurately measure $5 \mathrm{hmC}$, as this modification is commonly in low abundance in mammalian cells. HM450K arrays coupled with oxidative bisulphite provide a cost-effective representation of $5 \mathrm{hmC}$ distribution, at $\mathrm{CpG}$ sites with $5 \mathrm{hmC}$ levels $>\sim 10 \%$. However, $5 \mathrm{hmC}$ analysis is restricted to the genomic location of the probes, which is an important consideration as $5 \mathrm{hmC}$ modification is commonly enriched at enhancer elements. Finally, we show that the widely used hMeDIP-seq method provides an efficient genome-wide profile of $5 \mathrm{hmC}$ and shows high correlation with WG Bis/OxBis-seq $5 \mathrm{hmC}$ distribution in brain DNA. However, in cell line DNA with low levels of $5 \mathrm{hmC}$, hMeDIP-seq-enriched regions are not detected by WG Bis/OxBis or HM450K, either suggesting misinterpretation of $5 \mathrm{hmC}$ calls by hMeDIP or lack of sensitivity of the latter methods.

Conclusions: We highlight both the advantages and caveats of three commonly used genome-wide $5 \mathrm{hmC}$ profiling technologies and show that interpretation of $5 \mathrm{hmC}$ data can be significantly influenced by the sensitivity of methods used, especially as the levels of $5 \mathrm{hmC}$ are low and vary in different cell types and different genomic locations.

Keywords: DNA methylation, Methylome, Epigenetics, 5-Hydroxymethylation, HM450K Bis/OxBis, hMeDIP-seq

\footnotetext{
*Correspondence: s.clark@garvan.org.au; c.stirzaker@garvan.org.au

†Susan J. Clark and Clare Stirzaker contributed equally to this work

${ }^{1}$ Epigenetics Research Laboratory, Genomics and Epigenetics Division,

Garvan Institute of Medical Research, 384 Victoria Street, Darlinghurst,

Sydney, NSW 2010, Australia

Full list of author information is available at the end of the article
} 


\section{Background}

DNA cytosine methylation is one of the key epigenetic determinants of mammalian gene expression in normal development $[1,2]$ and disease [3]. DNA methylation is particularly dynamic during early embryonic development followed by "dynamic homeostasis" of the methylation landscape in normal functioning somatic cells [2]. The discovery that 5 -methylcytosine $(5 \mathrm{mC})$ can be oxidized to 5 -hydroxymethylcytosine $(5 \mathrm{hmC})$ by the ten-eleven translocation (TET) proteins has prompted wide interest in the potential roles of $5 \mathrm{hmC}$ in reshaping the mammalian DNA methylation landscape during early embryonic development $[4,5]$, during differentiation towards extraembryonic lineages $[6,7]$ and in metabolically active normal adult tissues $[8,9]$ and disease cells $[10]$. $5 \mathrm{hmC}$ levels vary substantially in somatic tissues [11] and the abundance and genomic distribution of $5 \mathrm{hmC}$ is dramatically altered during development $[8,12,13]$. Notably, $5 \mathrm{hmC}$ has been purported to play a key role as an intermediate in DNA demethylation; this may occur either passively during DNA replication [14], or actively through base excision repair of one or more oxidized intermediates [15, 16]. Other studies, however, regard $5 \mathrm{hmC}$ as a distinct epigenetic mark with a characteristic function independent of DNA demethylation [17-20]. The importance of the dynamic interplay between $5 \mathrm{mC}$ and $5 \mathrm{hmC}$ in maintaining normal DNA methylation patterns and gene expression and the causes and consequences of an imbalance are key questions yet to be answered.

Given the evidence that $5 \mathrm{hmC}$ plays a critical role in modulation of the DNA methylation landscape, it is essential to be able to distinguish $5 \mathrm{hmC}$ from $5 \mathrm{mC}$ and accurately detect and quantitate the levels of $5 \mathrm{hmC}$ at single-base resolution. In general, the levels of total $5 \mathrm{hmC}$ detected across the genome are approximately 14-fold lower than those of $5 \mathrm{mC}$ [21] although these levels vary substantially across tissue types: $5 \mathrm{hmC}$ is relatively abundant in brain tissues ( $0.15-0.6 \%$ of total nucleotides) [22, 23 ], but is an order of magnitude lower $(0.01-0.05 \%)$ in other mouse and human tissues [23-25]. In human cell lines, $5 \mathrm{hmC}$ abundance is at even lower levels $(\sim 0.007-$ $0.009 \%$ of total nucleotides) [26]. Such low and variable abundance means that the method of detection has to be highly sensitive and specific for the $5 \mathrm{hmC}$ modification.

Importantly, bisulphite sequencing, the "gold standard" for $5 \mathrm{mC}$ analysis, does not distinguish $5 \mathrm{mC}$ from $5 \mathrm{hmC}$, as both modified bases are resistant to conversion to uracil, in contrast to unmodified cytosine, which is converted to uracil (Fig. 1). This has precluded conventional bisulphite sequencing as a tool for $5 \mathrm{hmC}$ detection.

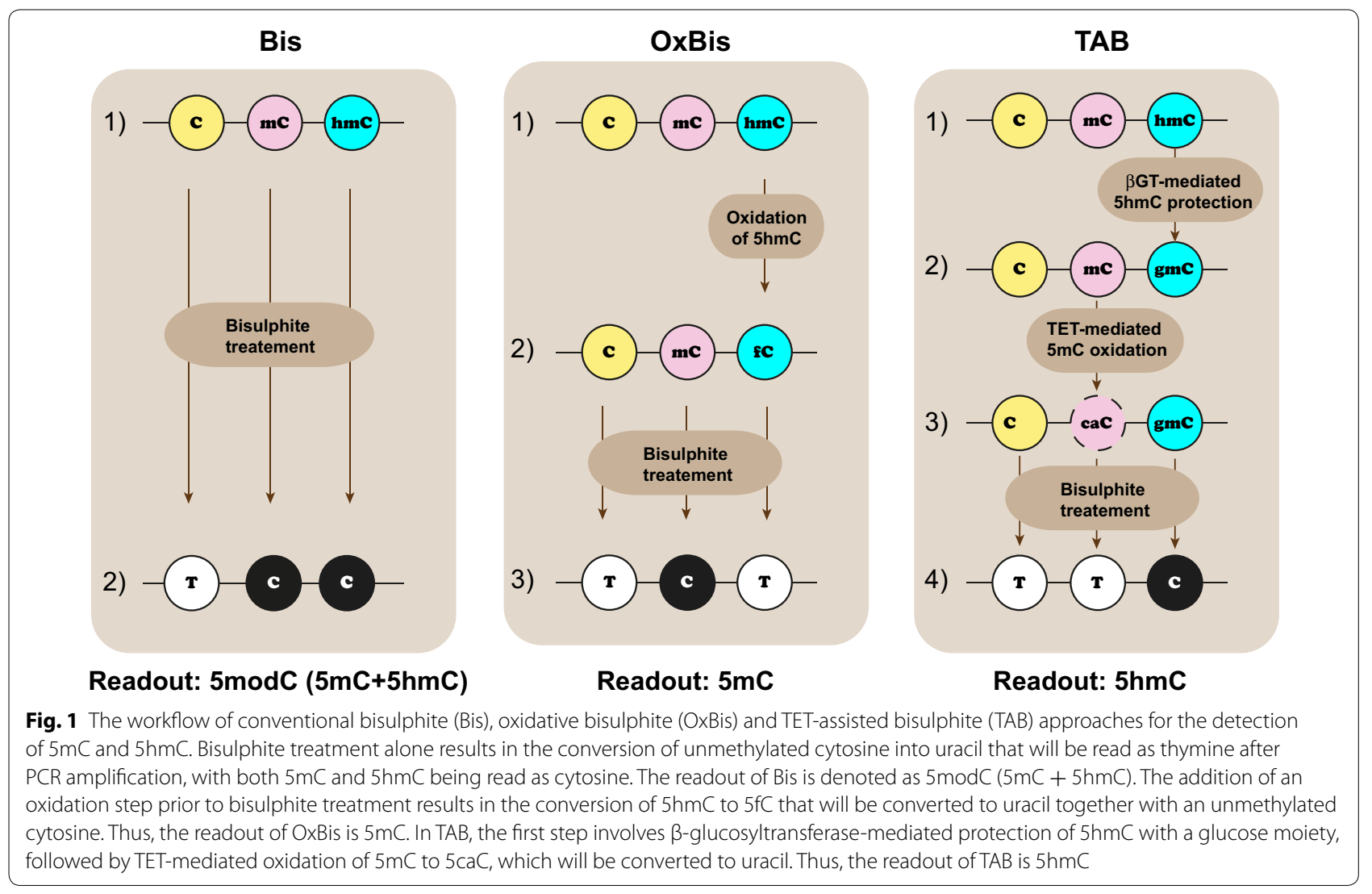


Several approaches for hydroxymethylation mapping have been developed over the recent years. These include capture-based techniques, such as antibody-based hydroxymethylated DNA immunoprecipitation followed by sequencing (hMeDIP-seq) [6,27, 28], and enrichment by hydroxymethyl selective chemical labelling (hMeSeal) [26]. These affinity-based methods have been widely used and provided the first genome-wide $5 \mathrm{hmC}$ profiles and biological insights of $5 \mathrm{hmC}[6,26-28]$. However, such approaches have relatively low resolution and cannot quantitatively determine $5 \mathrm{hmC}$ abundance in a singlebase resolution manner.

Single nucleotide $5 \mathrm{hmC}$ mapping approaches have also been developed. The two best-described approaches are whole-genome oxidative bisulphite in combination with conventional bisulphite sequencing (WG Bis/ OxBis-seq) [29] and TET-assisted bisulphite sequencing (TAB-seq) [30]. The principle of WG Bis/OxBis-seq relies on the specific oxidation of $5 \mathrm{hmC}$ by potassium perruthenate to form 5-formylcytosine (5fC) and/or 5-carboxylcytosine (5caC). $5 \mathrm{fC}$ and $5 \mathrm{caC}$ behave as unmethylated cytosines during bisulphite conversion. Therefore, the readout of OxBis-seq is specific for $5 \mathrm{mC}$ and does not contain the $5 \mathrm{hmC}$ fraction (Fig. 1a). Hence, subtraction of OxBis-seq readout $(5 \mathrm{mC})$ from the conventional Bis-seq readout $(5 \mathrm{mC}+5 \mathrm{hmC})$ evaluates the hydroxymethylated proportion at a single CpG site (Bisseq $(5 \mathrm{mC}+5 \mathrm{hmC})-$ OxBis-seq $(5 \mathrm{mC})=5 \mathrm{hmC})$. For clarity, we use $5 \operatorname{modC}$ to denote Bis-seq readout (i.e. total methylation: $5 \operatorname{modC}=5 \mathrm{mC}+5 \mathrm{hmC}$ ).

TAB-seq, on the other hand, gives a direct readout of $5 \mathrm{hmC}$. The first step of TAB reaction includes protection of $5 \mathrm{hmC}$ residues with a glucose moiety implemented by $\beta$-glucosyltransferase $(\beta-G T)$, whereas $5 \mathrm{mC}$ remains unprotected. Subsequent TET-mediated oxidation of $5 \mathrm{mC}$, but not "protected" $5 \mathrm{hmC}$, to $5 \mathrm{fC} / 5 \mathrm{caC}$ followed by bisulphite treatment results in a conversion of $5 \mathrm{fC} / 5 \mathrm{caC}$ and unmethylated cytosines to uracils, whereas hydroxymethylated cytosines remain as cytosines (Fig. 1b). Thus, while TAB-seq provides direct single nucleotide $5 \mathrm{hmC}$ profiling, OxBis-seq still requires conventional bisulphite (Bis-seq) to be performed in parallel to enable simultaneous $5 \mathrm{hmC}$ and $5 \mathrm{mC}$ single nucleotide readouts. These single nucleotide approaches also have been used for $5 \mathrm{hmC}$ interrogation on the HumanMethylation 450 (HM450K) BeadChip arrays [31, 32]. Similar to TABseq, HM450K-TAB provides a direct readout of $5 \mathrm{hmC}$, while HM450K Bis and OxBis arrays must be performed in parallel. Applying these single nucleotide approaches for whole-genome $5 \mathrm{hmC}$ mapping is ideal; however, the need for deep sequencing coverage for accurately resolving the $5 \mathrm{hmC}$ levels imposes restrictions and limitations on the widespread use of WG Bis/OxBis-seq and TABseq for mammalian genomes.

Here, we evaluate and compare three commonly used whole-genome $5 \mathrm{hmC}$ profiling approaches using WG Bis/OxBis-seq, HM450K arrays coupled with oxidative bisulphite (HM450K Bis/OxBis) and antibodybased immunoprecipitation of hydroxymethylated DNA (hMeDIP-seq). We also validate $5 \mathrm{hmC}$ at single nucleotide resolution using $\mathrm{TAB}$-seq of selected candidate genomic regions. The comparisons were made on adult frontal lobe cerebellum DNA, as the highest levels of $5 \mathrm{hmC}$ have been reported in adult brain DNA $[6,33]$. We highlight the advantages and caveats of $5 \mathrm{hmC}$ profiling methods and show that the interpretation of $5 \mathrm{hmC}$ data can be significantly influenced by the sensitivity of the method.

\section{Results}

\section{Hydroxymethylation profiling by whole-genome Bis-seq/ OxBis-seq}

To compare the different technologies for analysis of whole-genome $5 \mathrm{hmC}$ profiling, we first performed $5 \mathrm{hmC}$ profiling using WG Bis/OxBis-seq, which allows interrogation of both $5 \mathrm{hmC}$ and $5 \mathrm{mC}$ at single nucleotide resolution. Human frontal lobe DNA, spiked with M.SssI CpG-methylated $\lambda \mathrm{DNA}$ and fully hydroxymethylated $5 \mathrm{hmC}$ APC controls ("Methods" section), was treated with conventional bisulphite and oxidative bisulphite (CEGX TrueMethyl reagents), followed by library preparation and sequencing (Additional file 1: Table S1, Additional file 2: Figure S1; bisulphite conversion efficiency 98.30-99.76\%, 5hmC oxidation efficiency 99.33\%). The $5 \mathrm{mC}$ profile is a direct readout of Bis-seq, whereas the $5 \mathrm{hmC}$ profile is deduced by subtraction of the OxBis-seq readout from Bis-seq (Bis-OxBis).

Of the CpG sites considered significantly hydroxymethylated ( $p$ value $\leq 0.05$ : see "Methods" section), we found that the majority show $5 \mathrm{hmC}$ levels of $\sim 30 \%$ and high total methylation levels ( $5 \operatorname{modC})$ of $80-90 \%$ (Fig. 2a). To explore whether total methylation levels of $\mathrm{CpG}$ sites $(5 \operatorname{modC})$ are in proportion to $5 \mathrm{hmC}$ levels, we binned CpG sites based on their $5 \operatorname{modC}$ levels and plotted the distribution of $5 \mathrm{hmC}$ levels. The data reveal a linear relationship between total $\mathrm{CpG}$ methylation and $5 \mathrm{hmC}$, with 5 modC levels $<50 \%$, but a nonlinear relationship with 5 modC levels $>50 \%$ (Fig. 2 b). This observation is consistent with a dynamic interplay between $5 \mathrm{hmC}$ and $5 \mathrm{mC}$ at sites that display lower levels $(<50 \%)$ of total methylation or higher "plasticity" across the genome. In contrast, the more extensively methylated sites $(>50 \%)$ show a more stable and less "plastic" methylation state, due to the decreasing $5 \mathrm{hmC} / 5 \mathrm{mC}$ ratio. 


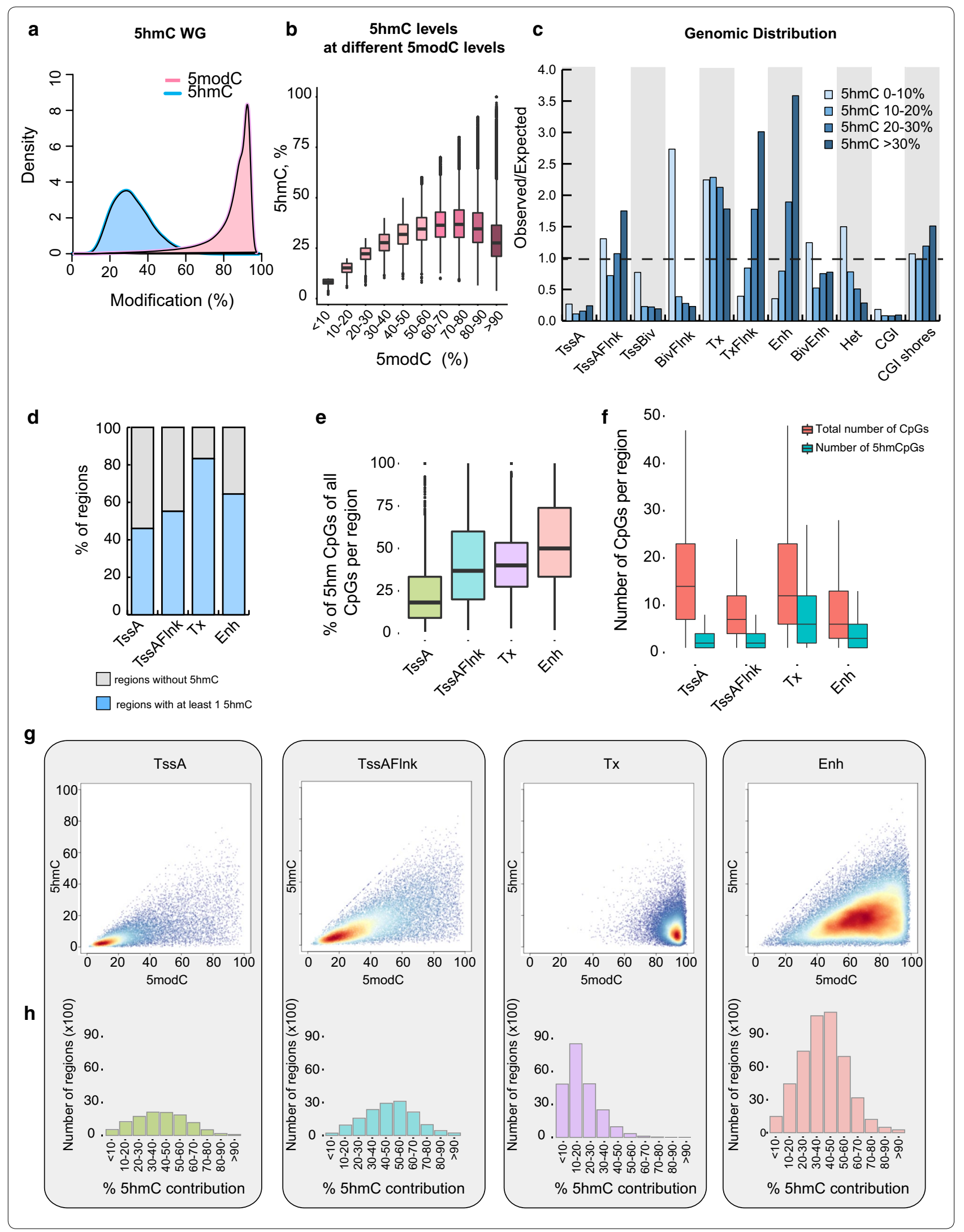


(See figure on previous page.)

Fig. 2 DNA methylation and hydroxymethylation profiling by whole-genome Bis-seq/OxBis-seq. a Methylation density plot showing the distribution of $5 \mathrm{hmC}$ and $5 \mathrm{modC}$ levels. Both $5 \mathrm{modC}$ and $5 \mathrm{hmC}$ density plots include $\mathrm{CpG}$ sites showing significant hydroxymethylation. b The relationship between the hydroxymethylated fraction and total methylation levels at each CpG site. CpG sites were binned into groups based on the total methylation levels, and the distribution of hydroxymethylation levels was calculated for each of these groups. c Bar plot showing observed over expected by chance enrichment of $\mathrm{CpGs}$ with different $5 \mathrm{hmC}$ levels at multiple genomic locations. Genomic regions comprise of Brain Frontal Lobe ChromHMM features as well as CpG islands and CpG island shores. $\mathbf{d}$ The percentage of genomic regions harbouring no hydroxymethylated CpG sites (grey) and those harbouring at least one hydroxymethylated CpG site (blue). e The proportion of hydroxymethylated CpGs of the total number of CpGs per genomic region. For each region, the total number of CpGs and number of hydroxymethylated CpGs were calculated (Additional file 2: Figure S1D). From that, for each region, the percentage of hydroxymethylated CpGs of all CpGs was calculated and distribution of those percentages was plotted. $\mathbf{f}$ The distribution of the total number of $\mathrm{CpG}$ sites and the number of hydroxymethylated $\mathrm{CpG}$ sites per genomic region. $\mathbf{g}$ The relationship between average total methylation $(5 \mathrm{modC})(x$-axis) and average hydroxymethylation $(5 \mathrm{hmC})(y$-axis) at different genomic regions. Each dot represents a single region. Hydroxymethylation levels of $\mathrm{CpG}$ sites that did not pass the statistical significance criteria were assigned to zero. $\mathbf{h}$ The hydroxymethylation contribution to the average total methylation at different genomic regions. For each genomic region, the percentage of hydroxymethylation contribution to the total methylation was calculated; the numbers of regions with the corresponding $5 \mathrm{hmC}$ contribution were plotted

Next, to investigate the genomic distribution of $5 \mathrm{hmC}$, we annotated $\mathrm{CpG}$ sites, according to the $5 \mathrm{hmC}$ levels, to Brain Frontal Lobe ChromHMM features [34, 35], as well as $\mathrm{CpG}$ islands and $\mathrm{CpG}$ island shores. We show that active and bivalent promoters (TssA and TssBiv) and CpG islands (CGI) are depleted of hydroxymethylated CpGs (Fig. 2c), coinciding with their prevalent unmethylated state. However, regions flanking active and bivalent promoters are enriched predominantly for CpGs with low $5 \mathrm{hmC}$ levels $(<10 \%)$ (Fig. $2 \mathrm{c}$ ), while transcribed regions (Tx), flanking transcribed regions (TxFlnk) and enhancers (Enh) possess the highest levels of $5 \mathrm{hmC}$ enrichment (Fig. 2c).

Finally, we asked whether the hydroxymethylated cytosines make a significant contribution to the overall total methylation levels $(5 \bmod C)$ at different genomic elements. To address this question, we focused on four genomic regions: active promoters, regions flanking active promoters, transcribed regions and enhancers (TssA, TssAFlnk, Tx, Enh, respectively). For each genomic region harbouring at least one $5 \mathrm{hmC}$ site (Fig. 2d), we calculated the percentage of hydroxymethylated CpGs of all CpGs and plotted the distribution (Fig. 2e). The analysis revealed $\sim 50 \%$ of $\mathrm{CpGs}$ at enhancers are hydroxymethylated (3 CpGs out of 6 on average), whereas only $\sim 15 \%$ of $\mathrm{CpG}$ sites at active promoters are hydroxymethylated (2 CpGs out of 13 on average) (Fig. 2e, f). To assess the contribution of $5 \mathrm{hmC}$ to the total methylation in each region, we plotted average $5 \mathrm{hmC}$ per region versus average total methylation (Fig. $2 \mathrm{~g}$ ) and calculated the number of regions with different $5 \mathrm{hmC}$ contributions (Fig. 2h). Despite the fact that only $15 \%$ of CpG sites at active promoters (TssA) are hydroxymethylated, $5 \mathrm{hmC}$ accounts for more than $\sim 50 \%$ of the total methylation at the vast majority of active promoters (Fig. 2g). Similarly, over half of enhancers and regions flanking active promoters show that $5 \mathrm{hmC}$ contributes significantly to the total average methylation (Fig. 2h). However, most of the transcribed regions have low $5 \mathrm{hmC}$ levels relative to the total average methylation (Fig. 2h). Overall, these data reveal that $5 \mathrm{hmC}$, when present, makes a substantial contribution to the total methylation levels at different genomic regions in brain DNA, highlighting the importance of discerning $5 \mathrm{hmC}$ from total methylation $(5 \operatorname{modC})$ in analysis of DNA methylomes from brain tissue.

\section{Methylation and hydroxymethylation profiling using Infinium HumanMethylation 450 methylation arrays} Infinium HumanMethylation450 BeadChip (HM450K) arrays have been widely used to study genome-wide $5 \mathrm{mC}$ DNA methylation profiles [36, 37], but more recently the technology has been adjusted to assess $5 \mathrm{hmC}[31,32]$. Here, we used the HM450K array in conjunction with OxBis to interrogate both $5 \mathrm{mC}$ and $5 \mathrm{hmC}$. Frontal lobe adult DNA was treated with conventional bisulphite and oxidative bisulphite reaction, respectively, followed by hybridization to HM450K arrays (HM450K Bis and HM450K OxBis). Arrays were performed in duplicate with technical replicates showing high correlation (Spearman correlation of 0.987 and 0.986 for HM450K Bis and HM450K OxBis replicates, respectively (Fig. 3a). Of 482,422 cytosines interrogated on the array, 175,000 CpG sites possess significant hydroxymethylation levels (i.e. probes showing a significant difference between Bis and OxBis: adjusted $p$ value $\leq 0.05$ : see "Methods" section).

We validated $5 \mathrm{hmC}$ levels detected by HM450K Bis/ OxBis using amplicon TAB-seq of selected loci: 4 regions showing significant hydroxymethylation and 3 regions that showed no significant hydroxymethylation by HM450K Bis/OxBis (Fig. 3c, Additional file 3: Figure S2). TET-mediated $5 \mathrm{mC}$ oxidation efficiency of M.SssI CpGmethylated $\lambda$ DNA was $98.74 \%$ and $\beta$-glucosyltransferasemediated protection of fully hydroxymethylated $5 \mathrm{hmC}$ pUC18 was 100\% ("Methods" section; Additional file 2: Figure S1). In parallel with TAB-seq, we performed 


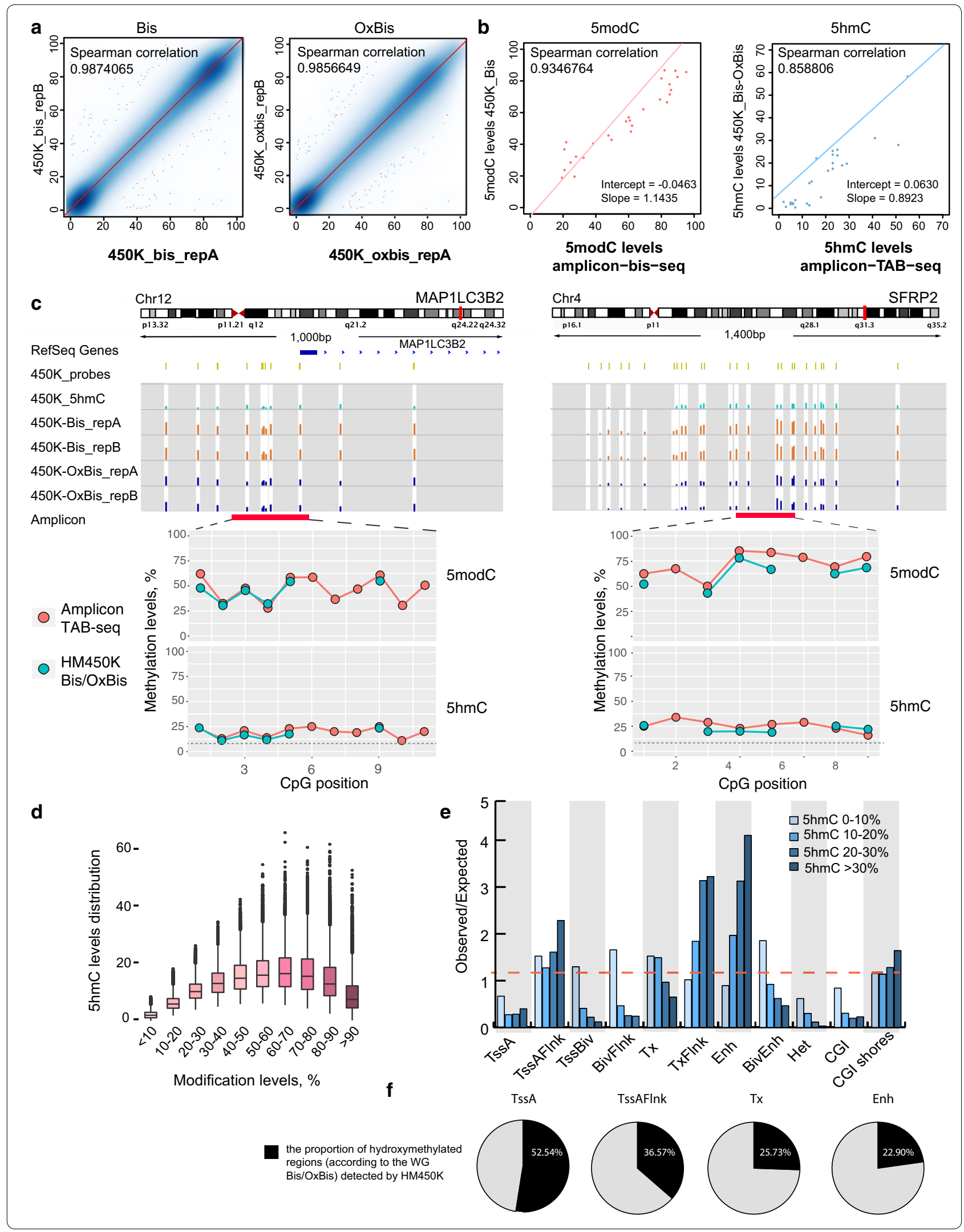




\begin{abstract}
(See figure on previous page.)
Fig. 3 DNA methylation and hydroxymethylation profiling by HM450K Bis/OxBis. a Scatter plots showing the high correlation between HM450K Bis (left) and HM450K OxBis (right) replicates. Each dot (smoothed) reflects each probe $5 \mathrm{modC}$ (left) or $5 \mathrm{mC}$ (right) levels detected by two technical replicates ( $x$ - and $y$-axes). Spearman's correlation 0.987406 (left) and 0.985664 (right), respectively. b, c 5hmC and 5 modC MiSeq amplicon validation of candidate loci using Bis- and TAB-Seq. b Scatter plots showing the correlation of $5 \mathrm{modC}$ (left) and $5 \mathrm{hmC}$ (right) levels between HM450K and amplicon Bis- and TAB-seq. Each dot represents single CpG site/probe. The pink and blue regression lines show the intercept and slope of the plots. c HM450K screen shots of candidate regions, showing 450K_5hmC, 450K_Bis, 450K_OxBis. Amplicon validation of these genomic regions shows agreement in total methylation ( $5 \mathrm{modC}$ ) and hydroxymethylation levels detected by HM450K Bis/OxBis and loci-specific Bis/TAB-seq, respectively. Red dots depict $5 \mathrm{modC}$ (top) and 5hmC (bottom) levels of each CpG site detected by loci-specific Bis-/TAB-seq, respectively. Green dots depict 5 modC (top) and $5 \mathrm{hmC}$ (bottom) levels of HM450K CpG probes on the array. $\mathbf{d}$ The relationship between hydroxymethylation and different levels of total methylation detected by HM450K. CpG probes were binned into groups based on the total methylation levels, and the distribution of hydroxymethylation levels was calculated for each of these groups. e Bar plot showing observed over expected by chance enrichment of CpGs with different $5 \mathrm{hmC}$ levels (0-10, 10-20, 20-30, >30\%) at multiple genomic locations; computationally derived chromatin segmentation (ChromHMM) of Brain Frontal Lobe genome, as well as CpG islands and CpG island shores. $\mathbf{f}$ Pie charts showing the proportion of genomic regions defined as hydroxymethylated by HM450K Bis/OxBis compared to the total number defined as hydroxymethylated according to the WG Bis/OxBis-seq. A region is considered hydroxymethylated if it contains $>1$ significantly hydroxymethylated $\mathrm{CpG}$ site
\end{abstract}

conventional bisulphite sequencing to validate total methylation levels of those loci. Since a subset of CpG sites in the selected regions correspond to HM450K probes, we were able to directly compare $5 \operatorname{modC}$ $(5 \mathrm{mC}+5 \mathrm{hmC})$ and $5 \mathrm{hmC}$ levels detected by HM450K Bis/OxBis with TAB-seq (Fig. 3c, Additional file 3: Figure S2A). Spearman's correlation of $5 \operatorname{modC}$ and $5 \mathrm{hmC} \mathrm{lev-}$ els across overlapping $\mathrm{CpG}$ sites from all loci sequenced shows 0.935 and 0.859 , respectively (Fig. 3b), confirming the high accuracy of cytosine hydroxymethylation detection by HM450K Bis/OxBis Infinium arrays. Notably, however, amplicon TAB-seq was more sensitive and could detect $5 \mathrm{hmC}$ levels $(\sim<10 \%)$ in regions that were not detected by HM450K (Additional file 3: Figure S2B), highlighting that that $5 \mathrm{hmC}$ levels need to be $>10 \%$ to be detected by HM450K Bis/OxBis.

Notably, loci-specific Bis- and TAB-seq, with sequencing coverage $\sim 50,000 \times$ and average of $13 \mathrm{CpG}$ sites per loci, can also be used to determine single-molecule $5 \operatorname{modC}(5 \mathrm{mC}+5 \mathrm{hmC})$ and $5 \mathrm{hmC}$ levels, respectively (Additional file 4: Figure S3). Interestingly, loci with intermediate 5 modC levels $(50-75 \%, x$-axis) predominantly consist of molecules with highly heterogeneous $5 \operatorname{modC}(\mathrm{A}$, purple and green dots) and $5 \mathrm{hmC}(\mathrm{B}$, green dots) single-molecule modification patterns, while loci with very low $(<25 \%, x$-axis) or very high $(>75 \%, x$-axis) levels of average $5 \bmod C$ methylation show more homogeneous patterns of $5 \operatorname{modC}(\mathrm{A}$, red and oranges dots, respectively) and $5 \mathrm{hmC}$ ( $\mathrm{B}$, red dots). This suggests that the vast majority of individual brain cells in the population with intermediate levels of $5 \operatorname{modC}$ methylation are not homogenous but display mosaic $5 \mathrm{hmC} / 5 \mathrm{mC}$ modification patterns.

Since HM450K methylation arrays interrogate only $1.8 \%$ of $\mathrm{CpG}$ sites and predominantly represent $\mathrm{CpG}$ island promoters [38], we wanted to explore whether the array results in biased $5 \mathrm{hmC}$ calling of CpGs with different total methylation levels or at different genomic locations. To address this, we binned HM450K CpG probes based on their $5 \operatorname{modC}$ levels and plotted the distribution of $5 \mathrm{hmC}$ levels at those $\mathrm{CpG}$ sites. The data reveal a linear relationship between total CpG methylation and $5 \mathrm{hmC}$, with $5 \operatorname{modC}$ levels $<50 \%$, but a nonlinear relationship with $5 \operatorname{modC}$ levels $>50 \%$, and notably CpGs with the highest $5 \operatorname{modC}$ levels $(90-100 \%)$ are depleted in the $5 \mathrm{hmC}$ fraction (Fig. 3d), in agreement with WG Bis/ OxBis-seq (Fig. 2b). The genomic distribution of $5 \mathrm{hmC}$ interrogated by HM450 also shows $5 \mathrm{hmC}$ observed/ expected enrichment at regions flanking Tss (TssAFlnk), flanking transcribed regions (TxFlnk) and enhancers (Enh), and depletion from active promoters (TssA) and CpG islands (CGI) (Fig. 3e) in agreement with WG Bis/ OxBis-seq (Fig. 2c). $5 \mathrm{hmC}$ was interrogated by HM450 at $\sim 53 \%$ of TssA and $37 \%$ of TssAFlnk; however, a smaller proportion of hydroxymethylated Enh and Tx regions (23 and $26 \%$, respectively) are detected by HM450K (Fig. 3f).

\section{Comparison of HM450K and whole-genome Bis-seq/ OxBis-seq for $5 \mathrm{hmC}$ mapping}

Next, we compared the performance of $5 \operatorname{modC}$ and $5 \mathrm{hmC}$ profiling using HM450K Bis/OxBis arrays with WG Bis/OxBis-seq. Of all HM450K probes, 207,125 had sequencing coverage greater than $10 \times$ in both WG Bisseq and OxBis-seq. Bis-seq and OxBis-seq values showed a high correlation between WG and HM450K data (Spearman's correlation 0.907 and 0.914 , respectively; Additional file 5: Figure S4). In addition, we determined the CpG sites common to both approaches with significant hydroxymethylation in both WG Bis/OxBis-seq and HM450K (42,537 probes) and compared the correlation of $5 \mathrm{modC}$ and $5 \mathrm{hmC}$ levels (Fig. 4a, Additional file 5: Figure S4). We found a good correlation between the platforms for $5 \operatorname{modC}(\mathrm{Bis})$ and $5 \mathrm{mC}$ (OxBis) methylation (Spearman's correlation 0.809 and 0.826 , respectively). 

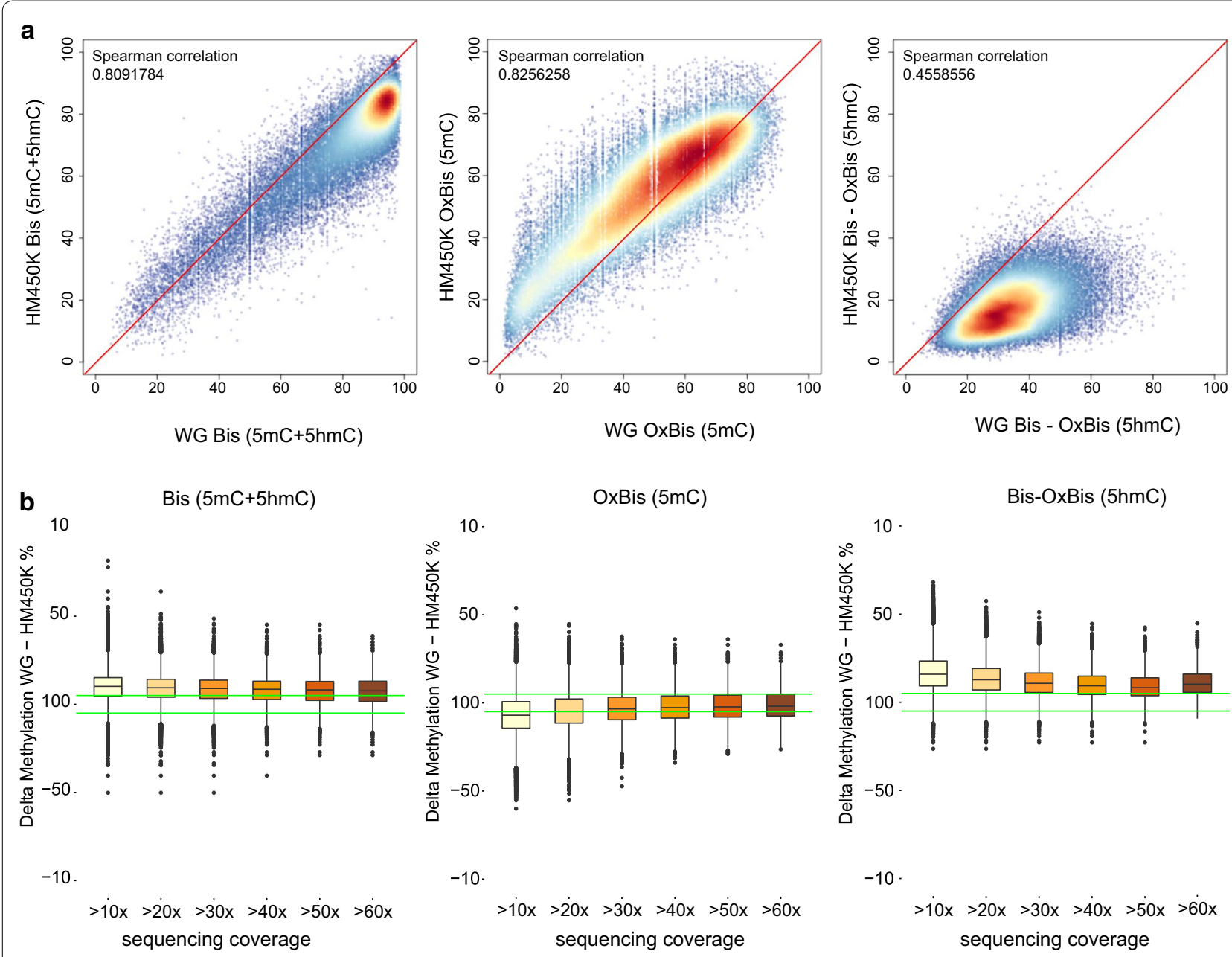

C

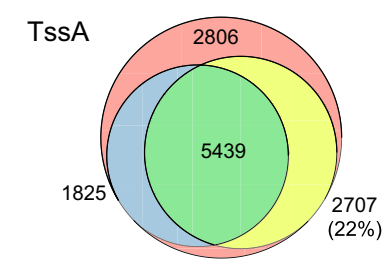

Tx

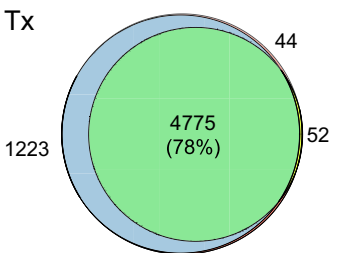

$\square$ regions without $5 \mathrm{hmC}$ on both HM450K and WG

$\square$ regions with $5 \mathrm{hmC}$ on HM450K only

$\square$ regions with $5 \mathrm{hmC}$ on WG only

$\square$ regions with $5 \mathrm{hmC}$ on both $\mathrm{HM} 450 \mathrm{~K}$ and WG d
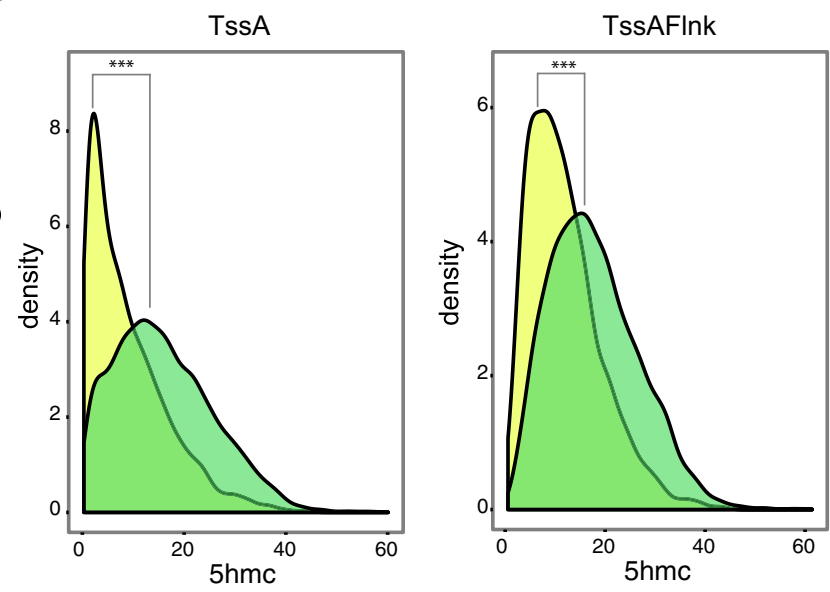

$\square$ regions with $5 \mathrm{hmC}$ on HM450K only

$\square$ regions with $5 \mathrm{hmC}$ on both $\mathrm{HM} 450 \mathrm{~K}$ and WG 
(See figure on previous page.)

Fig. 4 Comparative evaluation of HM450K Bis/OxBis and whole-genome Bis-/OxBis-seq for 5hmC profiling. a Scatter plots showing the correlation of $5 \mathrm{modC}$ (Bis, left), $5 \mathrm{mC}$ (OxBis, middle) and 5hmC (Bis-OxBis, right) between WG and HM450K Bis/OxBis across CpG sites $(n=42,537)$ considered as significantly hydroxymethylated by both approaches. Spearman's correlation is indicated on each scatter plot. $\mathbf{b}$ The agreement in $5 \mathrm{modC}, 5 \mathrm{mC}$ and $5 \mathrm{hmC}$ levels detected by WG and HM450K Bis/OxBis as a function of WG sequencing coverage. The difference in methylation calling is plotted along the $y$-axis for each bin with defined sequencing coverage indicated $(>10 x,>20 x,>30 x->60 x)$. Green lines indicate $\pm 5 \%$ difference in methylation value detected between approaches (WG-HM450K). c Venn diagrams show the overlap of hydroxymethylated regions between WG and HM450K Bis/OxBis. Genomic regions with at least one $\mathrm{CpG}$ probe and at least $10 \times$ WG sequencing coverage were chosen for this analysis (11,625 of 25,235 total TssA; 14,945 of 27,078 total TssA_Flnk; 22,249 of 26,707 total Tx; 47,082 of 73,063 total Enh). Of those, the number of regions with at least one hydroxymethylated CpG according to HM450K only, WG only or both approaches was calculated and overlap was plotted. d The distribution of maximal hydroxymethylation values at the regions identified as hydroxymethylated according to the HM450K only (yellow) and according to the both WG and HM450K (green). For the active promoters (TsSA), the median of the max (5hmC) distribution is 0.067 for the yellow group and 0.147 for the green group. For the regions flanking active promoters (TssA Flank), the median of the max(5hmC) distribution is 0.105 for the yellow group and 0.167 for the green group. The difference between the distributions of maximal $5 \mathrm{hmC}$ values between yellow and green groups of regions is statistically significant as determined by Kruskal-Wallis nonparametric test $(p<0.01)$

However, 5modC displays a greater discrepancy at high methylation levels (>80\%) with WG Bis-seq exceeding HM450K (Fig. 4a, Additional file 5: Figure S4). Such discordance between WG sequencing and HM450K at high 5 modC levels has been reported previously, highlighting the need for improved normalization strategies for HM450K analysis [39].

We next addressed the effect of sequencing coverage and found that increasing sequencing depth $(>10 \times-$ $>60 \times)$ does not improve the concordance between WG Bis-seq and HM450K Bis (Fig. 4b, left). However, increasing sequencing depth improves agreement between the methods for $5 \mathrm{mC}$ (OxBis) methylation comparison (Fig. 4b, middle). In contrast, correlation of $5 \mathrm{hmC} \mathrm{lev-}$ els between the two approaches does not improve with greater sequencing coverage (Fig. $4 \mathrm{~b}$, right) possibly due to the disagreement between WG and HM450K at high methylation levels.

We next determined whether the same genomic regions are defined as hydroxymethylated by both approaches. To this end, we took genomic regions covered by at least one HM450K probe, and at least $10 \times$ coverage on WG Bis/ OxBis-seq and only considered hydroxymethylation of those CpGs for the subsequent analysis. Of these regions, we defined hydroxymethylated regions $(>1 \times 5 \mathrm{hmC} \mathrm{CpG}$ site) according to HM450K only, WG Bis/OxBis only or both. We found that the vast majority of enhancers (73\%) and transcribed regions (78\%) are defined as hydroxymethylated by both approaches (Fig. 4c, green). The presence of hydroxymethylated regions defined by WG only (Fig. 4c, blue) is potentially due to the $5 \mathrm{hmC}$ undercalling of HM450K. Such agreement is lower for the active promoters and flanking regions (Fig. 4c, green), where a substantial proportion of regions (22 and 15\%, respectively) are not identified as hydroxymethylated on the WG (Fig. 4c, yellow). More detailed analysis revealed that regions identified as hydroxymethylated on HM450K only (yellow) possess lower levels of $5 \mathrm{hmC}$ compared to regions identified as hydroxymethylated by both approaches (Fig. 4d, green). This highlights that regions with lower $5 \mathrm{hmC}$ require higher sequencing coverage to detect $5 \mathrm{hmC}$ in the WG Bis/OxBis-seq data and therefore have not been detected as hydroxymethylated in the WG Bis/OxBis-seq data.

\section{Hydroxymethylation profiling by hMeDIP-seq}

One of the advantages of Bis/OxBis-seq and TAB-seq for hydroxymethylation mapping is that these methods quantitatively resolve $5 \mathrm{hmC}$ at a single nucleotide resolution; however, the depth of sequencing needed to yield meaningful results is generally not cost-effective. An alternative approach for genome-wide $5 \mathrm{hmC}$ profiling is antibody-based enrichment of $5 \mathrm{hmC}$, hMeDIP-seq, which is widely used due to its ease of use and cost-effectiveness. We performed hMeDIP on the same adult brain DNA (in duplicate), followed by library preparation and sequencing on HiSeq2500 to compare $5 \mathrm{hmC}$ calling with WG Bis/OxBis-seq and HM450K Bis/OxBis arrays.

To first compare signal enrichment between replicates, we binned the genome into 300-bp tiles and calculated $\log$ CPM values per bin. The data reveal a high correlation between replicates at high $\log \mathrm{CPM}$ values with overall Spearman's correlation of 0.756 (Fig. 5a). MACS2 peak calling resulted in 49,292 hMeDIP-seq peaks with enrichment at regions flanking active TSSes, enhancers and CpG island shores (Fig. 5b), similar to the distribution of 5hmC detected by WG (Fig. 2c) and HM450K Bis/OxBis (Fig. 3e). To next assess the specificity of antibody-based $5 \mathrm{hmC}$ detection, we compared the data with WG Bis/ OxBis-seq single nucleotide $5 \mathrm{hmC}$ mapping. We expanded each hMeDIP peak summit 150-bp up- and downstream (summits; Fig. 5c) and calculated average hydroxymethylation from WG Bis/OxBis-seq data and compared this to $5 \mathrm{hmC}$ at the regions not captured by hMeDIP-seq (gaps: Fig. 5c). The data revealed that hMeDIP peaks have significantly higher $5 \mathrm{hmC}$ levels $(>10 \%)$ compared to the 

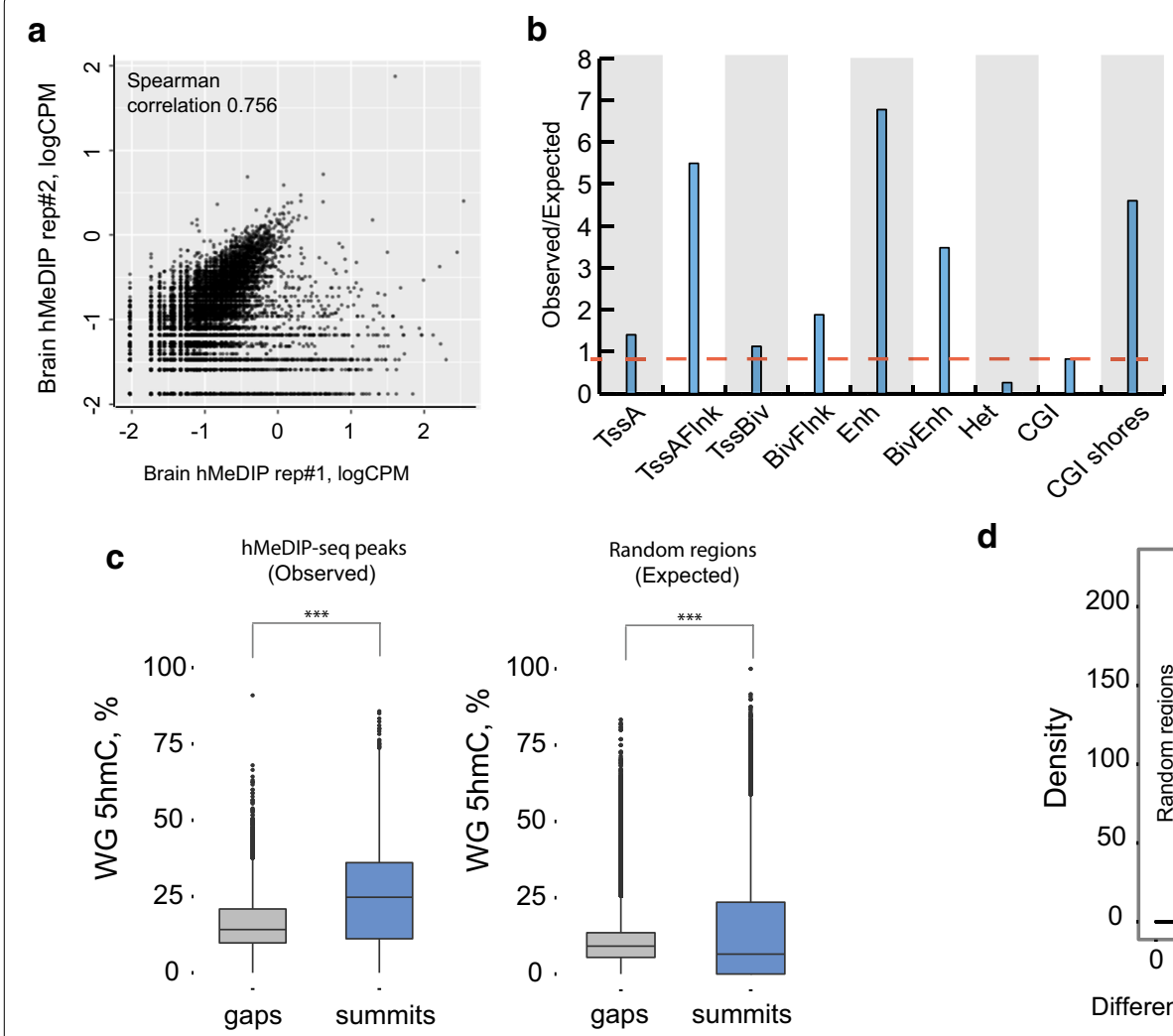

d

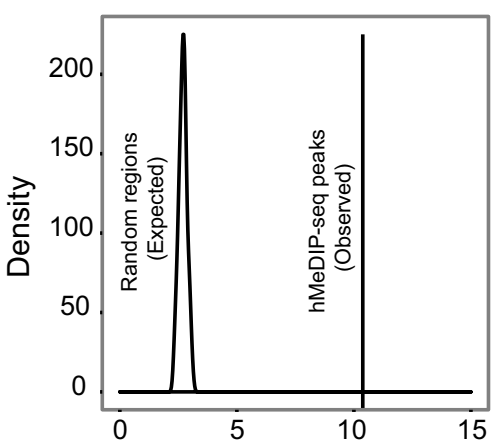

Difference of the mean of the $5 \mathrm{hmC}$ levels, \% (summits - gaps)
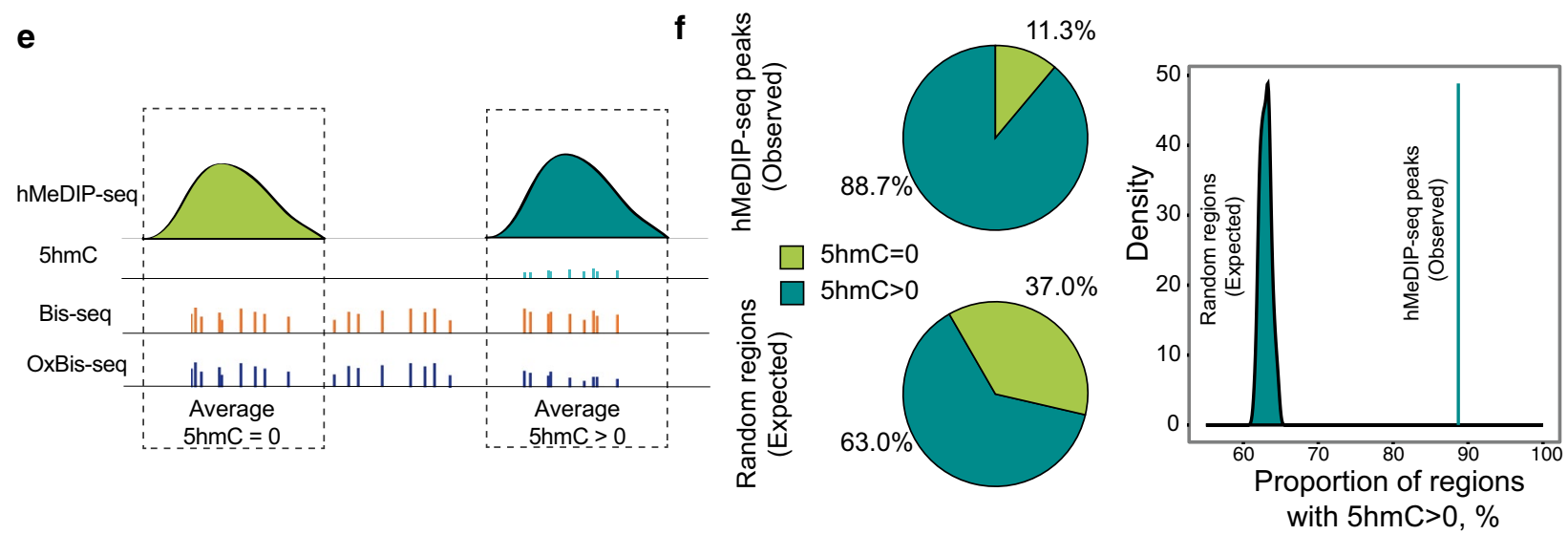

g

hMeDIP-seq peaks $(5 \mathrm{hmC}>0)$

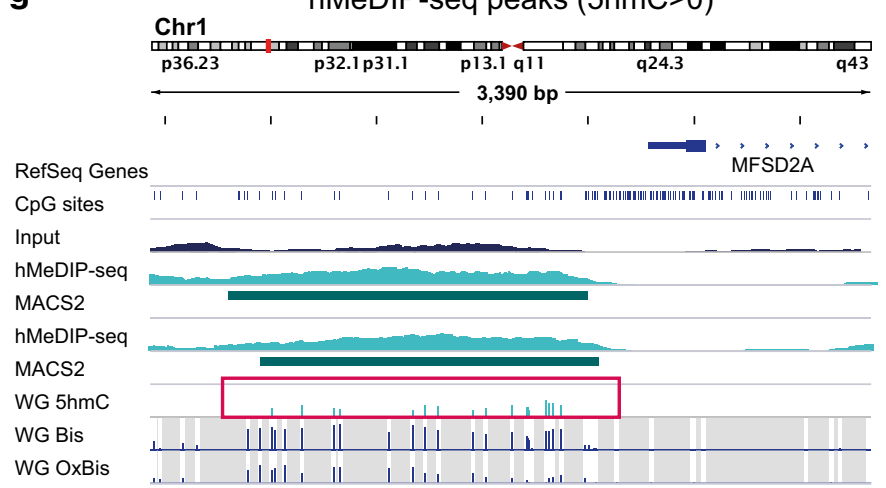

hMeDIP-seq peaks $(5 \mathrm{hmC}=0)$

Chr10
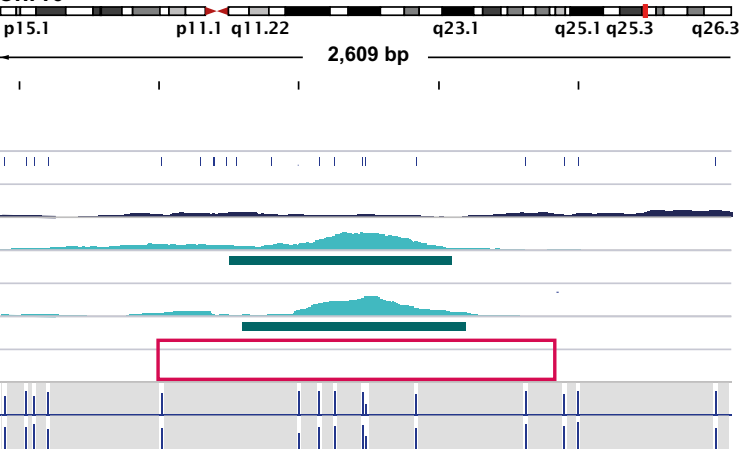


\begin{abstract}
(See figure on previous page.)
Fig. 5 hMeDIP-seq hydroxymethylation profiling in the brain. a Scatter plot showing the correlation between hMeDIP-seq replicates. For 300bp genomic tiles logCPM (count per million) values were calculated for each replicate. Each dot represents one genomic tile. b Bar plot showing observed over expected by chance enrichment of $5 \mathrm{hmC}$ peaks detected by hMeDIP-seq at multiple genomic locations; computationally derived chromatin segmentation (ChromHMM) of Brain Frontal Lobe genome, as well as CpG islands and CpG island shores. c, $\mathbf{d}$ Correlation of $5 \mathrm{hmC}$ profiling between WG Bis/OxBis and hMeDIP-seq. cThe distribution of WG-derived 5hmC levels at hMeDIP summits expanded \pm 150 bp and gaps between them (observed, left). Random permutation of hMeDIP summits and gaps and $5 \mathrm{hmC}$ levels distribution (expected, right). The differences between gaps and summits are statistically significant as determined by Kruskal-Wallis nonparametric test $(p<0.01)$. $\mathbf{d}$ The mean of the differences of $5 \mathrm{hmC}$ levels between hMeDIP-seq summits and gaps (observed) and the distribution of the mean for the permuted summits and gaps (expected). e Schematic representation of hMeDIP-seq peaks with no $5 \mathrm{hmC}$ detected by WG Bis/OxBis-seq (average $5 \mathrm{hmC}=0$ ) and with $5 \mathrm{hmC}$ detected by WG Bis/OxBis-seq (average $5 \mathrm{hmC}>0$ ). f Pie charts showing the proportion of real hMeDIP peaks (top) and randomly permuted hMeDIP peaks (bottom) with and without $5 \mathrm{hmC}$ detected by WG Bis/OxBis-seq. The distribution of the proportion of randomly permuted hMeDIP peaks with $5 \mathrm{hmC}$ (expected) and the actual proportion of hMeDIP peaks is shown on the right. For the analysis, hMeDIP-seq peaks with all associated CpGs having at least 10× WG coverage have been selected, which accounts for approximately 9000 peaks. $\mathbf{g}$ Genomic regions showing specific (left) and non-specific (right) hMeDIP-seq peaks
\end{abstract}

regions not captured by hMeDIP-seq (gaps; Fig. 5c, left). Random permutation of the hMeDIP peak summits and gaps does not result in the same trend (Fig. $5 \mathrm{c}$, right). The mean of the differences of $5 \mathrm{hmC}$ levels between hMeDIPseq peak summits and gaps (observed) is plotted alongside the distribution of the mean for the permuted summits and gaps (expected), showing the observed mean exceeding the expected mean (Fig. 5d), confirming the specificity of hMeDIP to $5 \mathrm{hmC}$. Interestingly, splitting hMeDIP signal based on the relative enrichment over input signal read counts ( $\operatorname{logFC}$, "Methods" section) into low, medium and high categories did not show differences in $5 \mathrm{hmC} \mathrm{lev-}$ els according to the WG Bis/OxBis (Additional file 6: Figure $\mathrm{S} 5 \mathrm{~B})$. This highlights the semi-quantitative nature of hMeDIP-seq.

Finally, to interrogate potential non-specific binding of hMeDIP, we focused on hMeDIP peaks with all CpG sites having at least $10 \times$ sequencing coverage in WG Bis-seq and OxBis-seq ("Methods" section). We separated hMeDIP-seq peaks with detected average $5 \mathrm{hmC}$ (5hmC $>0$, "Methods" section) and with no detected $5 \mathrm{hmC}(5 \mathrm{hmC}=0)$ in WG Bis/OxBis-seq (Fig. 5e). The relative abundance of hMeDIP peaks with detected average $5 \mathrm{hmC}>0$ is $88.7 \%$, revealing high specific hMeDIP binding (Fig. 5f, top). Random permutation of peaks resulted in a maximum of $65 \%$ of random peaks with $5 \mathrm{hmC}>0$, which is smaller than the observed value (88.7\%) (Fig. 5f, right). We noted that $11.3 \%$ of $5 \mathrm{hmC}$ was not detected by WG Bis/OxBis (Fig. 5f), suggesting that either higher sequencing coverage of WG Bis/OxBisseq is required and/or a degree of non-specific binding of hMeDIP (Fig. 5g, Additional file 6: Figure S5C). Together, the data show that hMeDIP-seq displays a high concordance with the WG Bis/OxBis-seq $5 \mathrm{hmC}$ profiling approach, even though $\sim 10 \%$ hMeDIP peaks represent potentially non-specific enrichment.

\section{Profiling low abundance $5 \mathrm{hmC}$ in cell line DNA}

In contrast to adult brain DNA with a known high abundance of $5 \mathrm{hmC}$, we next performed hMeDIP-seq on cancer cell line LNCaP DNA as cell lines are known to have low levels of $5 \mathrm{hmC}$ [18]. We observed less correlation (0.625) between replicates (Fig. 6a) than for the brain DNA (Fig. 5a). However, we show that the hMeDIP-seq peaks detected in prostate cancer cell line $\mathrm{LNCaP}$ show more correlation (0.384) with those identified in breast cancer cell line MCF7 (publically available: GSM1479831) (Fig. 6b, d) than brain DNA (0.123) (Fig. 6c), as expected between different DNA samples displaying low and high $5 \mathrm{hmC}$ content.

Next, we compared the performance of the single nucleotide 5hmC approaches (WG and HM450K Bis/ OxBis) for brain and LNCaP DNA. Both single nucleotide $5 \mathrm{hmC}$ profiling approaches show the distribution of the $5 \mathrm{hmC}$ signal centred around zero with similar proportion of positive and negative values (Fig. 6e, g); however, the $5 \mathrm{hmC}$ signal in brain tissue is shifted towards positive values in both approaches (Fig. 6e, g). Since $5 \mathrm{hmC}$ is a subtraction of OxBis from the Bis signal, the distribution of $5 \mathrm{hmC}$ signal around zero reflects, per se, the error rates of both approaches. Further, the proportion of significantly hydroxymethylated CpGs of all CpGs (see "Methods" section) in the brain is 10 times greater than that of LNCaP cells (27.7 and 2.5\% respectively), as detected by WG Bis/OxBis-seq (Fig. 6f). Importantly, $\mathrm{HM} 450 \mathrm{~K}$ Bis/OxBis did not detect any significant $5 \mathrm{hmC}$ in LNCaP cells (Fig. $6 \mathrm{~h}$ ), in contrast to the $>35 \%$ of CpGs in the brain found to be hydroxymethylated by $\mathrm{HM} 450 \mathrm{~K}$ Bis/OxBis (Fig. 6h).

To rule out the possibility that the absence of $5 \mathrm{hmC}$ calling by the single nucleotide approaches is driven by the lack of sequencing coverage in WG Bis/OxBis and limitations of sensitivity of the HM450K array, we 

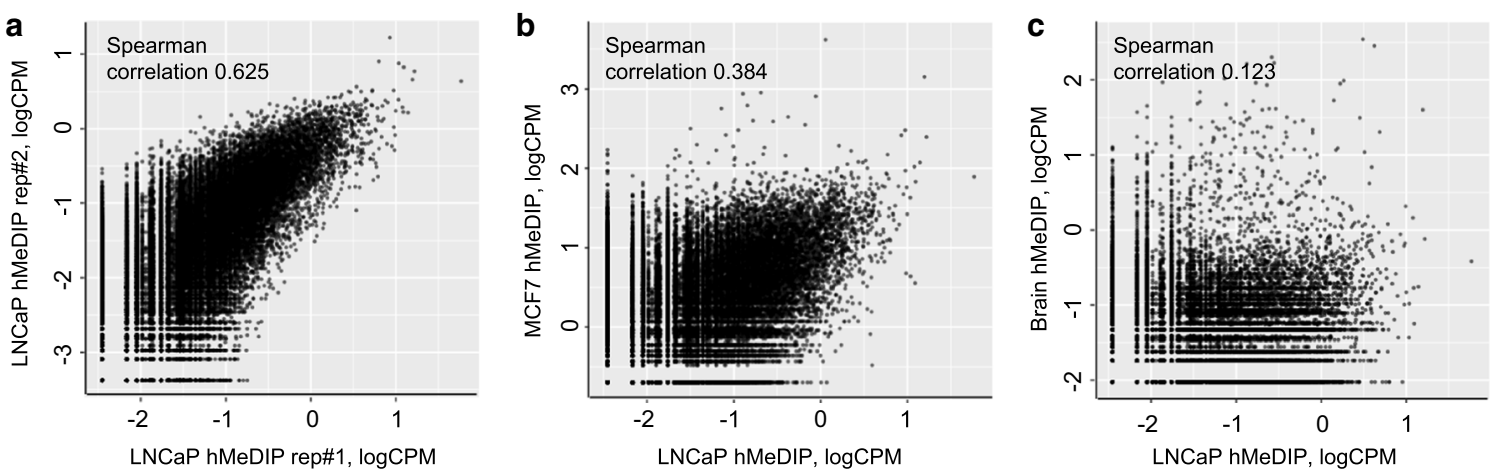

d

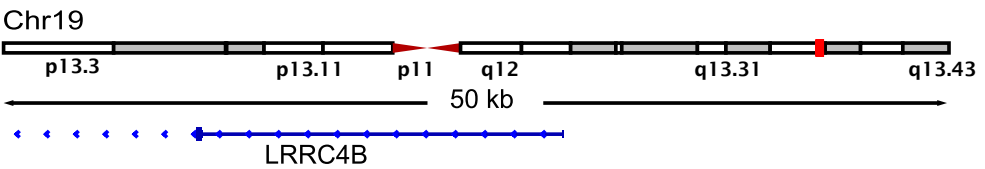

RefSeq Genes

CpG sites

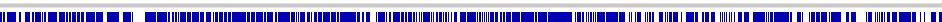

$\mathrm{CpG}$ islands

LNCaP hMeDIP-seq rep\#1

LNCaP hMeDIP-seq rep\#2

MCF7 hMeDIP-seq

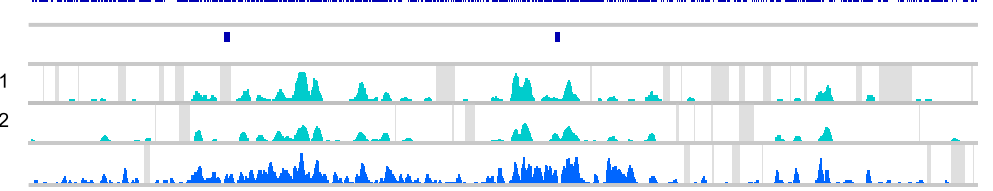

e
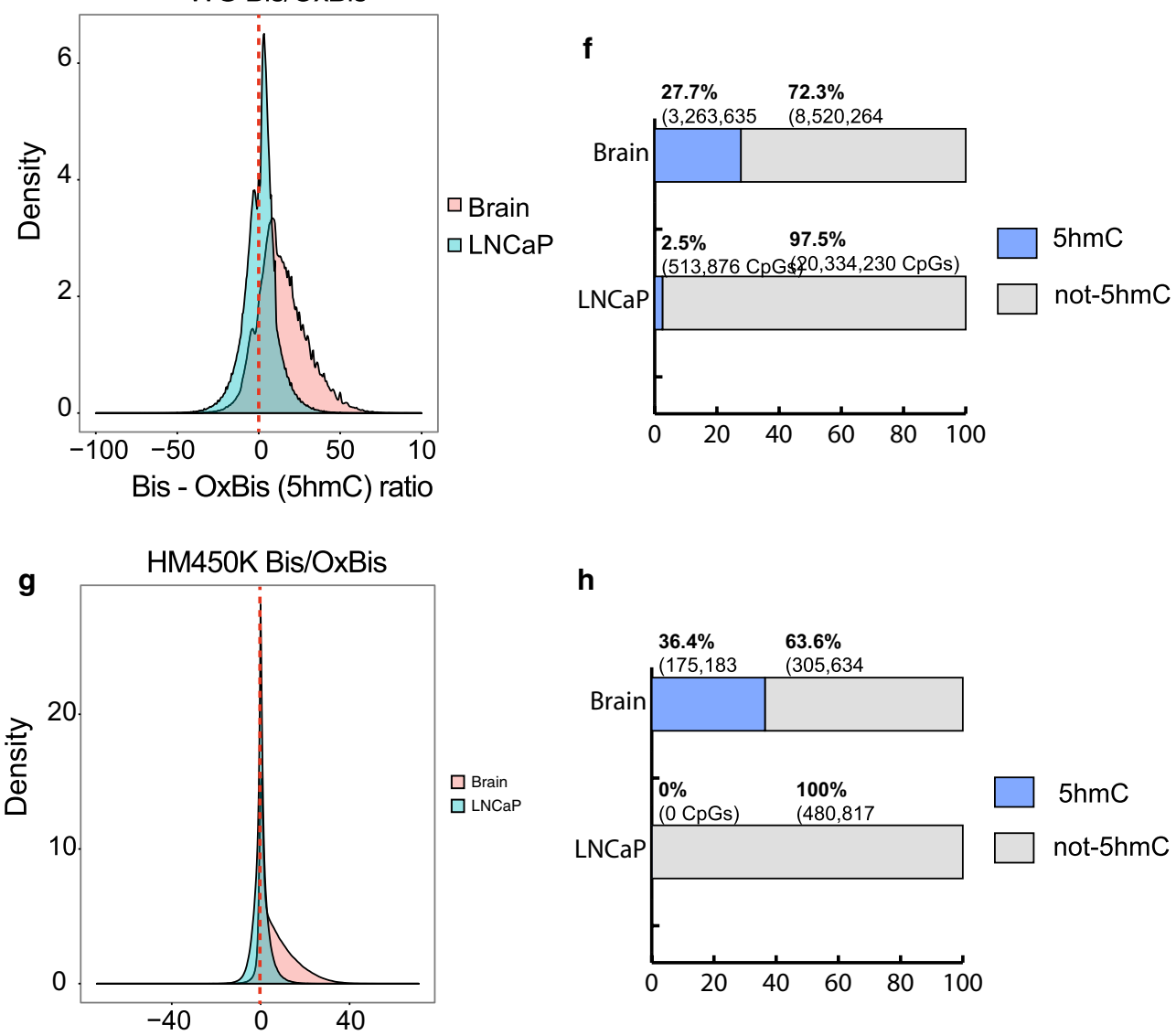

h

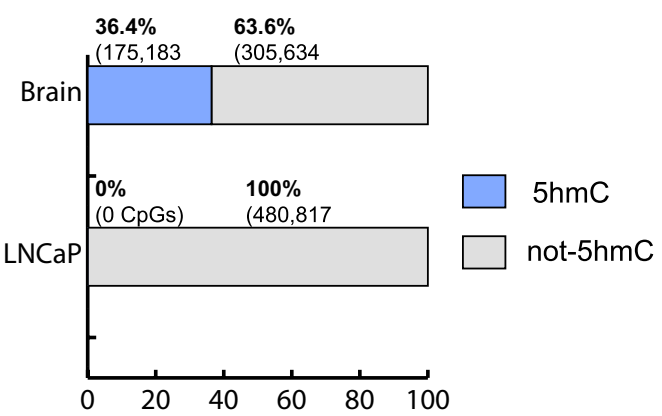

Bis - OxBis $(5 \mathrm{hmC})$ ratio 
(See figure on previous page.)

Fig. 6 hMeDIP-seq hydroxymethylation profiling in cell line DNA. a Scatter plot showing the correlation between hMeDIP-seq replicates in LNCaP cells. For each genomic tile from one replicate the average enrichment score for the second replicate was calculated. Each dot represents one genomic tile. b Scatter plot showing the correlation between hMeDIP-seq in LNCaP cells and public hMeDIP-seq in MCF7 cells. c Scatter plot showing the correlation between hMeDIP-seq in LNCaP cells and brain hMeDIP-seq. d Genomic region showing the correspondence of hMeDIP-seq replicates in LNCaP cells as well as MCF7 cells. e Density plot showing the distribution of $p_{\text {Bis }}-p_{\text {OxBis }}$ values in the Brain versus LNCaP WG Bis/OxBis data. $\mathbf{f}$ The percentages of significantly hydroxymethylated CpGs of all CpGs with at least $10 \times$ coverage on the WG Bis/OxBis in the Brain versus LNCaP. $\mathbf{g}$ Density plot showing the distribution of $p_{\text {Bis }}-p_{\text {OxBis }}$ values in the Brain versus LNCaP HM450K Bis/OxBis data. $\mathbf{h}$ The percentages of significantly hydroxymethylated CpGs of all CpGs with at least 10x coverage on the HM450K Bis/OxBis in the Brain versus LNCaP

performed loci-specific TAB-seq with high $(\sim 50,000 \times)$ sequencing coverage. We chose regions that were detected by hMeDIP-seq (Additional file 7: Figure S6). While loci-specific TAB-seq showed $5 \mathrm{hmC}$ levels of $<2-4 \%$ (Additional file 7: Figure S6), a proportion of the levels are comparable to the technique error rate $(\sim 1.8 \%$, "Methods" section), suggesting potential non-specificity of hMeDIP-seq in genomes with low $(<2-4 \%) 5 \mathrm{hmC}$ abundance.

These findings highlight that for DNA of high $5 \mathrm{hmC}$ abundance ( $5 \mathrm{hmC}$ levels $>10 \%$ ) hMeDIP-seq shows high specificity. However, in the absence or presence of very low levels of $5 \mathrm{hmC}(<\sim 2-4 \%)$, such as in cell line DNA, hMeDIP-seq signals cannot be used as the representative of the actual $5 \mathrm{hmC}$ distribution without further validation.

\section{Discussion}

For more than two and half decades, DNA methylation studies in higher organisms have relied on the use of bisulphite conversion technologies to study DNA methylation $[38,40]$. However, it is now understood that these technologies do not distinguish between 5-methylcytosine $(5 \mathrm{mC})$ and 5-hydroxymethylcytosine $(5 \mathrm{hmC})$ [41, 42]. Since the discovery of 5-hydroxymethylcytosine and its potential role in modulating the DNA methylation landscape, there has been significant interest in defining the genome-wide distribution of $5 \mathrm{hmC}$. Importantly, there is a critical need to distinguish $5 \mathrm{hmC}$ from $5 \mathrm{mC}$ and accurately detect and quantitate the levels of $5 \mathrm{hmC}$ at single-base resolution. A number of strategies have been developed to map $5 \mathrm{hmC}$, including affinitybased approaches $[6,27,28]$ and chemical modification approaches that allow single nucleotide resolution analyses $[29,30,43]$. In general, the abundance of total $5 \mathrm{hmC}$ detected across the genome is approximately tenfold to 100 -fold lower [18] than that of $5 \mathrm{mC}$, posing technical challenges for many approaches. Here, we have assessed three $5 \mathrm{hmC}$ genome-wide profiling approaches, using whole-genome bisulphite/oxidative bisulphite sequencing (WG Bis/OxBis-seq), Infinium HumanMethylation450 BeadChip arrays coupled with OxBis (HM450K Bis/OxBis) and antibody-based immunoprecipitation of hydroxymethylated DNA (hMeDIP-seq).
WG Bis/OxBis-seq approach enables analysis of the relationship between $5 \mathrm{mC}$ and $5 \mathrm{hmC}$ at the single nucleotide level. We were able to identify hydroxymethylated genomic regulatory regions (Fig. 2c, d), in particular at enhancer regions (Fig. 2e), highlighting the importance of taking $5 \mathrm{hmC}$ into consideration, especially when studying DNA methylation dynamics at distal regulatory regions in brain DNA. Interestingly, the vast majority of CpG sites with moderate modification levels possess the highest hydroxymethylation proportion compared to the more densely methylated CpGs that generally possess lower $5 \mathrm{hmC}$ signal (Fig. 2b). In addition, genomic regions with moderate methylation levels show mosaic $5 \mathrm{hmC} / 5 \mathrm{mC}$ single-molecule methylation patterns. This observation is consistent with the hypothesis that $5 \mathrm{hmC}$ is prevalent at dynamic CpG sites showing higher "plasticity" across the genome [44]. Thus, the ability to discriminate $5 \mathrm{mC}$ and $5 \mathrm{hmC}$ on a genome-wide scale allows the contribution of $5 \mathrm{hmC}$ to total methylation to be elucidated, and to identify where regions are more dynamic or stably methylated.

Single nucleotide whole-genome $5 \mathrm{hmC}$ profiling is theoretically the most ideal approach to interrogating unbiased distribution of $5 \mathrm{hmC}$ across the genome. However, the use of WG approaches is restricted due to its high cost. To accurately assess $5 \mathrm{hmC}$ levels at single nucleotide resolution, $5 \mathrm{hmC}$ profiling requires higher sequencing depth, compared to $5 \mathrm{mC}$ methylation profiling since hydroxymethylation levels are more than a magnitude lower than DNA methylation levels [45]. As an alternative, HM450 BeadChip arrays have been modified to allow the detection of $5 \mathrm{hmC}$ separate from $5 \mathrm{mC}$, used in conjunction with both oxidative [31] and TET-assisted bisulphite conversion [32]. Due to the fact that HM450K interrogates only $1.8 \%$ of all CpG sites in the human genome, $5 \mathrm{hmC}$ detection is restricted to the regions interrogated on the array. Using HM450K Bis/OxBis, we were able to detect hydroxymethylation at $\sim 50 \%$ of promoters and $\sim 20 \%$ of enhancers, which were identified as hydroxymethylated according to the WG Bis/OxBis-seq (Fig. 3f). However, since enhancers possess the highest enrichment of $5 \mathrm{hmC}$ among all genomic elements, a substantial proportion of potentially $5 \mathrm{hmC}$-dependent functional genomic elements are not detected by HM450K. 
Importantly, the newly released Illumina EPIC array covers over $850,000 \mathrm{CpG}$ sites, including $>90 \%$ of the CpGs from the HM450 BeadChip and an additional 413,743 CpGs. The additional probes improve the coverage of regulatory elements, including enhancers, and therefore, this will offer significantly enhanced coverage of $5 \mathrm{hmC}$ enriched genomic elements.

Overall, detection of hydroxymethylation at different genomic regions is highly concordant between approaches (Fig. 4c). However, HM450K Bis/OxBis possess a tendency of $5 \mathrm{hmC}$ signal underestimation, driven by CpG sites with extreme levels of total methylation, highlighting the necessity of improved normalization approaches of the array signal. This results in the "loss" of a significant number ( 20\%) of hydroxymethylated regions at different regulatory elements. On the other hand, a subset of promoters and flanking regions are identified as hydroxymethylated on HM450K Bis/OxBis only (Fig. 4d). Those regions possessed lower levels of $5 \mathrm{hmC}$ and therefore require higher sequencing coverage highlighting the higher sensitivity of the HM450 compared to the WG at a given sequencing coverage.

In contrast, antibody-based enrichment hMeDIPseq of $5 \mathrm{hmC}$ has been widely used as a relatively easy and cost-effective approach. Overall, we observed a good agreement between hMeDIP-seq and WG Bis/ OxBis-seq $5 \mathrm{hmC}$ signal as well as high concordance in the patterns of $5 \mathrm{hmC}$ genomic distribution. Moreover, we showed that, despite the presence of $5 \mathrm{hmC}$ signal in the hMeDIP-seq in cell line DNA, neither HM450K Bis/OxBis nor WG Bis/OxBis-seq and loci-specific TAB-seq were able to detect any significant $5 \mathrm{hmC}$ signal. This finding, together with the $5 \mathrm{hmC}$ profiling in adult brain DNA, suggests that in the presence of high levels of $5 \mathrm{hmC}$, hMeDIP-seq is reliable, whereas in the absence or presence of very low levels of $5 \mathrm{hmC}$, such as cell line DNA, hMeDIP-seq signal is potentially subject to misinterpretation of $5 \mathrm{hmC}$ distribution. An alternative affinity-based approach, hMeSeal, has been used for cost-effective whole-genome $5 \mathrm{hmC}$ profiling [24, 26]. While it has been shown to be a reliable and sensitive approach $[18,26]$ and successfully performed on low input DNA material [46], a detailed comparative analysis would be required to assess its performance compared to single nucleotide $5 \mathrm{hmC}$ profiling techniques on a wholegenome scale.

\section{Conclusions}

In this study, we provide a detailed comparison of three genomic $5 \mathrm{hmC}$ profiling approaches. $5 \mathrm{hmC}$ profiling with WG Bis/OxBis-seq provides the most comprehensive quantitative overview of $5 \mathrm{mC}$ and $5 \mathrm{hmC}$ distribution across the whole genome in cells displaying higher levels of $5 \mathrm{hmC}$, such as brain tissue. HM450K Bis/OxBis provides a user-friendly, high-throughput and affordable approach for cells that display higher levels of $5 \mathrm{hmC}$, but will miss regions not present on the array and shows undercalling of $5 \mathrm{hmC}$ signal driven by $\mathrm{CpGs}$ with high methylation. Finally, hMeDIP-seq is a widely used and accepted approach due to its ease of use and cost-effectiveness. However, it is semi-quantitative, does not allow single nucleotide resolution and has the potential for non-specific enrichment in DNA displaying low $5 \mathrm{hmC}$ levels. Overall, we find a high correlation of hMeDIP with both WG Bis/OxBis and HM450K Bis/OxBis. Ultimately the method of choice for whole-genome profiling of $5 \mathrm{hmC}$ will depend on the abundance of $5 \mathrm{hmC}$, number and quantity of DNA samples, cost consideration, bioinformatics expertise and the question being addressed.

\section{Methods \\ DNA samples}

Adult Brain Frontal Lobe genomic DNA from a single donor was obtained from Banksia Scientific Company (Bulimba, Australia, Cat No D1234035). Genomic DNA from the prostate cancer cell line $\mathrm{LNCaP}$ was extracted using QIAamp DNA Mini kit (Qiagen, USA). LNCaP prostate cells were cultured as described previously [47].

\section{Whole-genome bisulphite and oxidative bisulphite sequencing}

Adult brain genomic DNA was sheared to an average size of $800 \mathrm{bp} ; 200 \mathrm{ng}$ was used for the bisulphite (Bis) and oxidative bisulphite (OxBis) reactions. To assess the efficiency of potassium perruthenate-mediated oxidation of $5 \mathrm{hmC}$ and the behaviour of $5 \mathrm{mC}$, genomic DNA was spiked with 5-hydroxymethylated 338-bp PCR product of APC genomic locus and M.SssI $\lambda$ DNA, respectively (described in "Spike-in controls for WG Bis/OxBis and TAB-seq" section). The bisulphite and oxidative bisulphite reactions were performed according to the manufacturer's instructions (CEGX TrueMethyl ${ }^{\circledR}$ WG user guide v2). Library preparation and indexing were also carried out as described (CEGX TrueMethyl ${ }^{\circledR}$ WG). Library quality was assessed with the Agilent 2100 Bioanalyzer using the high-sensitivity DNA kit (Agilent, CA, USA). DNA was quantified using the KAPA Library Quantification kit by quantitative PCR (KAPA Biosystems). Paired-end 150-bp sequencing was performed for each library on the Illumina HiSeqX platform using the HiSeq $\mathrm{X}^{\mathrm{TM}}$ Ten Reagent Kit v2.

\section{Infinium HM450K bisulphite and oxidative bisulphite beadchip arrays}

DNA was treated in separate aliquots with CEGX Bis and OxBis reagents according to the manufacturer's 
specifications (CEGX TrueMethyl ${ }^{\circledR}$ _UGuide). $2 \mu \mathrm{g}$ DNA was first sheared to $10 \mathrm{~kb}$ and then purified on BioRad ${ }^{\circledR}$ P6 Micro-Bio spin column. Two aliquots were subjected to oxidation and two to mock oxidation prior to the Bis treatment (see CEGX protocol). Following the Bis reaction, the samples were re-quantified using the Qubit ssDNA assay. A minimum sample concentration of $20 \mathrm{ng} / \mu \mathrm{l}$ was required for the next step of the $450 \mathrm{~K}$ process to ensure $7 \mu \mathrm{l}$ of bisulphite-converted sample contains 140-160 ng of bisulphite-converted DNA. $7 \mu \mathrm{l}$ of recovered TrueMethyl template was used in the HM450K protocol with $1 \mu \mathrm{l}$ of $0.4 \mathrm{~N} \mathrm{NaOH}$ (see Infinium Methylation assay). All subsequent steps were completed according to Illumina Infinium HM450K beadarray chip instructions.

\section{Hydroxymethylation profiling by hMeDIP-seq}

DNA was sonicated with Covaris to produce fragments in size range of $300-500$ bp. Prior to hMeDIP procedure, Illumina adaptors were ligated to $(5 \times 1 \mu \mathrm{g})$ of fragmented DNA as described in the TruSeq LT DNA Sample Preparation kit, Illumina. The hMeDIP assay was performed according to the manufacturer's instructions (Active Motif, hMeDIP, Cat No 55010). Briefly, $3 \times 1 \mu \mathrm{g}$ of fragmented adapter-ligated DNA was spiked with $50 \mathrm{ng}$ of either unmethylated, $5 \mathrm{mC}$ methylated or $5 \mathrm{hmC}$ hydroxymethylated 338-bp PCR product of APC genomic locus. The DNA was denatured for $10 \mathrm{~min}$ at $95{ }^{\circ} \mathrm{C}$ and immunoprecipitated overnight at $4{ }^{\circ} \mathrm{C}$ with $4 \mu \mathrm{l}$ of $5 \mathrm{hmC}$ polyclonal antibody (Active Motif Cat No 55010). To allow selective enrichment of immune-captured DNA fragments, the mixture was incubated with $25 \mu \mathrm{l}$ of Protein G magnetic beads for $2 \mathrm{~h}$ at $4{ }^{\circ} \mathrm{C}$ prior to washing all unbound DNA fragments. The bound hydroxymethylated DNA was eluted, treated with proteinase $\mathrm{K}$ and purified by Phenol/chloroform/isoamyl alcohol extraction and ethanol precipitation. The specificity of the hMeDIP assay was validated by $\mathrm{qPCR}$ of the unmethylated, methylated and hydroxymethylated spike-in APC controls and dot blots.

\section{Bisulphite and TET-assisted bisulphite treatment for amplicon sequencing Bisulphite reaction}

Bisulphite reaction was performed using EZ DNA Methylation-Gold Kit (Zymo Research, USA, Cat No D5005) according to the manufacturer's instructions. M.SssI $\lambda$ DNA $(5 \mathrm{mC})$ was used as a spike-in control (described in "Spike-in controls for WG Bis/OxBis and TAB-seq" section) to assess the efficiency of bisulphite conversion reaction.

\section{TET-assisted bisulphite treatment}

TET-assisted bisulphite treatment was performed using the 5hmC TAB-seq Kit (WiseGene, USA, Cat No K001) according to the manufacturer's instruction. Briefly, $1 \mu \mathrm{g}$ genomic DNA was sonicated to the size of approximately $2 \mathrm{kbp}$ according to the manufacturer's instructions. After sonication, DNA was spiked with $10 \mathrm{ng}$ (1\%) M.SssI $\lambda$ DNA $(5 \mathrm{mC})$ control and $10 \mathrm{ng}(1 \%) 5 \mathrm{hmC}$ pUC18 control DNA. The $\beta$-GT-based reaction was performed at $37^{\circ} \mathrm{C}$ for $1 \mathrm{~h}$ and the DNA purified using QIAquick PCR Purification kit (Qiagen, USA, Cat No 28106) according to the protocol and eluted in $27 \mu$ water. The eluted DNA was split into two separate reactions to ensure no more than 300 ng DNA per TET1-based oxidation reaction. The TET1 oxidation reaction was performed at $37{ }^{\circ} \mathrm{C}$ for $1 \mathrm{~h}$, followed by the treatment of $1 \mu \mathrm{l}$ of proteinase $\mathrm{K}$ $(20 \mathrm{mg} / \mathrm{ml})$ at $50{ }^{\circ} \mathrm{C}$ for $1 \mathrm{~h}$. The oxidized DNA was purified using QIAquick PCR purification kit (Qiagen, USA, Cat No 28106) and eluted in $50 \mu \mathrm{l}$ water. TET1-oxidized DNA was then bisulphite-treated above using the EZ DNA Methylation-Gold kit (Zymo Research, USA Cat No D5005) as described in the protocol. Post-bisulphite conversion PCR amplification was performed in triplicate (4 ng/PCR); PCRs were pooled and purified using Wizard $^{\circledR}$ SV Gel and PCR Clean-Up System (Promega, USA, Cat No A9282). Library prep was performed following the instructions as per the Illumina TruSeq DNA sample prep kit (Cat No FC-121-2001) described below.

\section{Spike-in controls for WG Bis/OxBis and TAB-seq}

Spike-in controls were used to assess the efficiency of the bisulphite, oxidative bisulphite and TET-assisted bisulphite reactions.

\section{M.SssI $\lambda D N A(5 m C)$}

We generated an in vitro $\mathrm{CpG}(5 \mathrm{mC})$-methylated $\lambda \mathrm{DNA}$ control to (1) assess the bisulphite conversion efficiency of unmethylated cytosines (in a non-CpG context) to uracils and (2) to assess the efficiency of TET-mediated oxidation of $5 \mathrm{mC}$ in TAB-seq reaction. Unmethylated $\lambda \mathrm{DNA}$ (Promega, USA, Cat No D1521) was sonicated to the average size of approximately $2 \mathrm{kbp}$ according to the manufacturer's instructions. $3 \mu \mathrm{g}$ of sonicated DNA was used in methylation reaction using $4 \mathrm{U}$ of $\mathrm{CpG}$ methyltransferase M.SssI (New England Biolabs, USA, Cat No M0226S) in the presence of $640 \mu \mathrm{M}$ SAM. Methylation reaction was allowed to proceed at $37{ }^{\circ} \mathrm{C}$ for $2 \mathrm{~h}$ and was stopped by heating at $65{ }^{\circ} \mathrm{C}$ for $20 \mathrm{~min}$. CpG-methylated $\lambda$ DNA was purified using QIAquick PCR purification kit according to the protocol. After the completion of TAB reaction, 290-bp fragment of $\lambda \mathrm{DNA}$ was amplified using following primers:

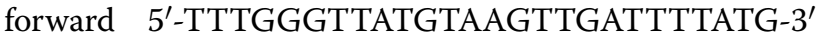


and reverse 5'-CACCCTACTTACTAAAATTTACACC-3' (Additional file 8: Table S2). The PCR product was $3^{\prime}$-adenylated and ligated into the pGEM-T-easy plasmid (Promega, USA, Cat No A1360), followed by MiSeq amplicon sequencing.

For WG Bis/OxBis, the efficiency of bisulphite conversion was $98.30 \%$ for Bis and $99.76 \%$ for OxBis. For TAB-seq, the efficiency of bisulphite conversion was 99.58\% and TET-mediated $5 \mathrm{mC}$-to-T oxidation efficiency was $98.74 \%$ (Additional file 2: Figure S1C, D). The efficiency of M.SssI $\lambda$ DNA CpG methylation was assessed by clonal Sanger sequencing after the completion of the conventional bisulphite reaction, which was performed alongside.

\section{$5 \mathrm{hmC}$ pUC18 control}

To assess the efficiency of $\beta$-GT-mediated protection of $5 \mathrm{hmC}$, an in vitro hydroxymethylated pUC18 control was generated. 1.64-kbp region of pUC18 plasmid was amplified in the presence of 5-hydroxymethyl-dCTP, 5hmdCTP (Zymo Research, USA, Cat No D1045) using following primers: forward 5'-GCAGATTGTACTGAGAGTGC- $3^{\prime}$ and reverse 5'-TGCTGATAAATCTGGAGCCG-3' (Additional file 8: Table S2). After the completion of TAB reaction, 190-bp fragment of 1.64kbp $5 \mathrm{hmC}$ pUC18 control was amplified using the following primers: forward 5'-GTAGATTGTATTGAGAGTGT-3' and reverse 5'-TACCCAACTTAATCGCCTTG-3' (Additional file 8: Table S2), followed by the clonal Sanger sequencing as described for the M.SssI $\lambda$ DNA control. Due to the contamination of $5 \mathrm{hmdCTP}$ with unmodified $5 \mathrm{dCTP}$, the actual degree of hydroxymethylation of $5 \mathrm{hmC}$ pUC18 control had to be assessed by clonal Sanger sequencing after the completion of the conventional bisulphite reaction, which was performed alongside. The $\beta$-GT-mediated protection was $\sim 100 \%$ as estimated using $5 \mathrm{hmC}$-to-T conversion efficiency of $5 \mathrm{hmC}$ pUC18 control in TAB reaction normalized to that in the conventional bisulphite reaction (Additional file 2: Figure S1D).

\section{5hmC APC control}

To assess the efficiency of potassium perruthenate $\left(\mathrm{KRuO}_{4}\right)$-mediated oxidation of $5 \mathrm{mC}$ in the OxBis reaction, Illumina TruSeq DNA adapters were ligated to the commercially available $5 \mathrm{hmC}$ APC PCR product of APC genomic locus (Active Motif, USA, Cat No 55008). Briefly, $1 \mu \mathrm{g}$ of $5 \mathrm{hmC}$ APC control was used for the end repair, A-tailing and ligation of Illumina adapter according to the Illumina instructions. After the completion of the OxBis reaction, $5 \mathrm{hmC}$ APC PCR product was amplified using PCR primer cocktail supplied by Illumina followed by the clonal Sanger sequencing as described for the M.SssI $\lambda$ DNA control (Additional file 2: Figure S1B). The $\mathrm{KRuO}_{4}$-mediated $5 \mathrm{mC}$-to- $5 \mathrm{caC} /(\mathrm{T})$ oxidation efficiency was $99.33 \%$ as estimated using $5 \mathrm{hmC}$-to- $5 \mathrm{caC} /(\mathrm{T})$ conversion efficiency of $5 \mathrm{hmC}$ pUC18 control in OxBis reaction normalized to that in the conventional bisulphite reaction.

\section{MiSeq amplicon $T A B$ sequencing}

Validation of candidate regions was performed on bisulphite- and TET-assisted bisulphite-treated DNA described above. First, PCRs were performed on a temperature gradient between 50 and $62{ }^{\circ} \mathrm{C}$ to achieve optimal amplification temperature. Each PCR was checked on an agarose gel for specific PCR products. To test for the amplification bias, we used the following bisulphitetreated control DNA: (1) human genomic blood DNA (Roche Cat No 11691112001) (unmethylated control DNA); (2) serological DNA from Chemicon (100\% methylated control); (3) 50:50 mix of Roche and serological DNA under three different concentrations of $\mathrm{MgCl}_{2}$. For each PCR, optimal temperature and $\mathrm{MgCl}_{2}$ concentrations were determined as described previously [48].

PCRs of Adult Brain and LNCaP DNA (bisulphite or TET-assisted bisulphite treated) were performed in triplicate using optimized conditions (Additional file 8: Table S2). PCRs were pooled and purified using Wizard SV and PCR Clean-Up System (Promega, USA, Cat No A9282) and quantitated by Qubit. Library prep was performed following the instructions as per the Illumina TruSeq DNA sample prep kit (Cat No FC-121-2001). Briefly, $1000 \mathrm{ng}$ of pooled amplicon input DNA was used for each library preparation. End repair, A-tailing and ligation of Illumina adapter to pooled PCR library were performed according to the Illumina instructions. After PCR clean-up, the library was quantified by Qubit, diluted to $10 \mathrm{nM}$ according to Qubit, and accurately quantitated by KAPA SYBR FAST Universal qPCR (KAPA Biosystems, USA, Cat No KK4835) before being sequenced on the Illumina MiSeq ${ }^{\mathrm{TM}}$ sequencer (Illumina, CA, USA).

\section{Data analysis}

Data processing and alignment was performed using inhouse computational pipelines. Statistical analyses were conducted in the R statistical software.

\section{Whole-genome Bis/OxBis sequencing data}

Bisulphite reads were aligned to the human genome using version 1.2 of an internally developed pipeline, publicly available for download from http://github.com/ astatham/Bisulphite_tools. Briefly, adaptor sequences and poor-quality bases were removed using TrimGalore (version 0.2.8, http://www.bioinformatics.babraham. ac.uk/projects/trim_galore/) in paired-end mode with 
default parameters. Bismark v0.8.326 was then used to align reads to hg19 using the parameters "-p 4 -bowtie2 -X 1000 -unmapped -ambiguous -gzip -bam”. PCR duplicates were removed using Picard v1.91 (http://broadinstitute.github.io/picard). Count tables of the number of methylated and unmethylated bases sequenced at each CpG site in the genome were constructed using bismark_methylation_extractor with the parameters “- $p$-no_overlap -ignore_r2 4-comprehensive -merge_ non-CpG -bedgraph - counts - report -gzip -buffer_size 20G". The adult brain Bis and OxBis libraries had a total of 517,530,911 and 489,841,771 reads, respectively. Both libraries passed basic quality control checks with $89 / 90 \%$ alignment rate and $18 \times / 16 \times$ mean coverage for adult brain Bis/OxBis, respectively.

To assess the level of $5 \mathrm{hmC}$ at a CpG locus, we compared the number of retained cytosines in Bis experiment to that in OxBis experiment. More specifically, we assumed that the number of retained cytosines follows a binomial distribution $N C \sim \operatorname{Binomial}(N, p)$, where $N$ is the coverage and $p$ is the proportion of modified cytosines. For the Bis experiment $p_{\text {Bis }}=p_{5 \mathrm{mC}}+p_{5 \mathrm{hmC}}$ is the proportion of both $5 \mathrm{mC}$ and $5 \mathrm{hmC}$ modifications, whereas for OxBis experiment $p_{\mathrm{OxBis}}=p_{5 \mathrm{mC}}$ is the proportion of $5 \mathrm{mC}$ modifications. Thus, to assess the proportion of $5 \mathrm{hmC}$ modifications we used proportion test, as implemented in R's prop.test() function, to estimate the difference between $p_{\text {Bis }}$ and $p_{\text {OxBis }}$ given $N C_{\text {Bis }}, N_{\text {Bis }}$, $N C_{\text {OxBis }}$ and $N_{\text {OxBis }}$.

Average coverage for Bis-seq and OxBis-seq was $18 \times$ and $16 \times$, respectively (Additional file 1 : Table S1). Before applying proportion test, we filtered out $\mathrm{CpG}$ loci having less than 10x coverage in either Bis or OxBis dataset. This reduced the number of tested CpG loci from 28,269,977 to $11,783,899$ for adult brain data. CpGs with statistically significant difference between $\mathrm{Bis}$ and $\mathrm{OxBis}(p$ value $<0.05, p_{\text {Bis }}-p_{\text {OxBis }}>0$ ) were considered as significantly hydroxymethylated resulting in 3,263,635 CpG sites. Only these CpG sites are considered in the subsequent analyses. For the calculations of the average hydroxymethylation per region, we discarded CpGs with $p$ value $\geq 0.05$ and $p_{\text {Bis }}-p_{\text {OxBis }}>10 \%$ as well as CpGs with $p_{\text {Bis }}-p_{\text {OxBis }}<0$ and imputed CpGs with $p$ value $\geq 0.05$ and $0<p_{\text {Bis }}-p_{\text {OxBis }}<10 \%$ to zero.

We performed power calculations using R's power. prop.test() function to determine power as a function of coverage in both Bis and OxBis experiments.

\section{HM450K Bis/OxBis data}

Two replicates of adult brain samples per treatment condition, Bis or OxBis, were profiled on Illumina's HumanMethylation450K array [37]. The raw data were preprocessed and background normalized with
Biconductor minfi package [49] using preprocessIllu$\operatorname{mina}(\ldots$, bg.correct $=$ TRUE, normalize $=$ "controls", reference $=1$ ) normalization function. We used the limma Bioconductor package $[49,50]$ to perform differential methylation analysis between Bis and OxBis treatments to determine levels of $5 \mathrm{hmC}$. We only considered probes for which there was a reliable methylation readout (detection $p$ value $<0.01$ ) in all four samples. We then transformed $\beta$-values into $M$-values using logit transformation: $M=\log \left(\frac{\beta}{1-\beta}\right)$. (To avoid extreme $M$-values, the $\beta$-values were capped at 0.01 and 0.99.) Standard limma workflow with unpaired contrast was then applied to computed $M$-values to call differentially methylated probes between Bis and OxBis and thus to determine levels of $5 \mathrm{hmC}$. This analysis resulted in 175,183 probes having significant hydroxymethylation $(p$ value $<0.05$, $p_{\text {Bis }}-p_{\text {OxBis }}>0$ ).

\section{TAB-seq data}

Paired-end fastq files were obtained for each library and aligned to hg19 using bwa-meth (http://github.com/ brentp/bwa-meth, arXiv:1401.1129). Downstream analysis was performed using the "ampliconAnalysis" function of the $\mathrm{R}$ package aaRon (http://github.com/astatham/ aaRon). Data quality was checked by assessing the number of reads obtained and the bisulphite conversion efficiency per amplicon and per sample. All samples had high bisulphite conversion efficiency $>98 \%$ and amplicons had $>10,000 \times$ coverage. Percent hydroxymethylation/methylation at each $\mathrm{CpG}$ site was calculated.

\section{hMeDIP sequencing data}

Sequenced reads from hMeDIP immunoprecipitated and input control human brain samples were mapped to the reference human genome (hg19) with bowtie v.1.1.0 [51], allowing up to three mismatches. Reads mapping to multiple locations and/or deemed as PCR duplicates were filtered out. Reads were extended $300 \mathrm{bp}$ and overlapped with the 300-bp tiling of the human genome to create a table of counts to be used for statistical modelling. Bins with low total number of reads (less than 20) across the three samples were removed from the analysis. Standard analysis flow as implemented in the edgeR Bioconductor package [52] was then applied to contrast read counts in hMeDIP samples to the input control. The library normalization step as implemented in calcNormFactors() was omitted and dispersion was set to 0.01 . The same analysis was repeated for regions of $300 \mathrm{bp}$ centred on summits of broad peaks identified with MACS2 algorithm [53]. Summit regions from two hMeDIP replicates were merged resulting in 137,598 regions used for the analysis. Of those, 137,373 regions had $p$ value $<0.05$ and $\operatorname{logFC}>0$ (with the smallest logFC 1.391). Regions with FDR $<0.1$ 
and $\log \mathrm{FC}>0$ (smallest $\log \mathrm{FC} 1.391$ ) were called as having $5 \mathrm{hmC}$ modification resulting in 21,553 marked regions for summit-centred analysis. For the specificity analysis, we selected only hMeDIP-seq regions with all CpGs per region having at least $10 \times$ coverage in the WG Bis/ OxBis resulting in 7563 regions. Next, we calculated average hydroxymethylation (as described in "Whole-genome Bis/OxBis sequencing data" section) per each region and separated into two categories: average $5 \mathrm{hmC}>0$ and $5 \mathrm{hmC}=0$. For LNCaP, hMeDIP replicates were merged resulting in 240,216 regions used for the analysis.

\section{Genome annotation \\ Genomic locations}

Genomic coordinates (hg19) of CpG islands were obtained from UCSC genome browser. Genomic coordinates of $\mathrm{CpG}$ island shores were derived by taking $\pm 2-\mathrm{kb}$ flanking regions around $\mathrm{CpG}$ islands.

\section{ChromHMM annotations}

A bed-formatted annotation file of chromatin states was downloaded from the Encode Roadmap (http://egg2. wustl.edu/roadmap/data/byFileType/chromhmmSegmentations/ChmmModels/coreMarks/jointModel/final/ E073_15_coreMarks_dense.bb) [34, 35]. We used hypergeometric testing to determine statistical significance of overlap between regional hypomethylated probes and the above functional annotations of the genome.

\section{Additional files}

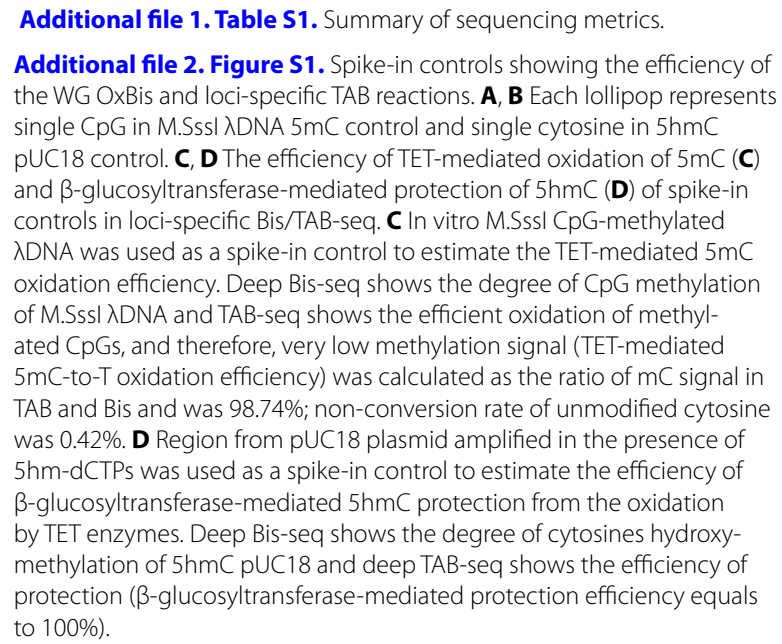

Additional file 2. Figure S1. Spike-in controls showing the efficiency of the WG OxBis and loci-specific TAB reactions. A, B Each lollipop represents single CpG in M.SssI $\lambda$ DNA $5 \mathrm{mC}$ control and single cytosine in $5 \mathrm{hmC}$ pUC18 control. C, D The efficiency of TET-mediated oxidation of $5 \mathrm{mC}$ (C) and $\beta$-glucosyltransferase-mediated protection of $5 \mathrm{hmC}$ (D) of spike-in controls in loci-specific Bis/TAB-seq. C In vitro M.Sssl CpG-methylated $\lambda D N A$ was used as a spike-in control to estimate the TET-mediated $5 \mathrm{mC}$ oxidation efficiency. Deep Bis-seq shows the degree of $\mathrm{CpG}$ methylation of M.SssI XDNA and TAB-seq shows the efficient oxidation of methylated $\mathrm{CpGs}$, and therefore, very low methylation signal (TET-mediated $5 \mathrm{mC}$-to-T oxidation efficiency) was calculated as the ratio of $\mathrm{mC}$ signal in TAB and Bis and was 98.74\%; non-conversion rate of unmodified cytosine was $0.42 \%$. D Region from pUC18 plasmid amplified in the presence of $5 \mathrm{hm}-\mathrm{dCTPs}$ was used as a spike-in control to estimate the efficiency of $\beta$-glucosyltransferase-mediated $5 \mathrm{hmC}$ protection from the oxidation by TET enzymes. Deep Bis-seq shows the degree of cytosines hydroxymethylation of 5hmC pUC18 and deep TAB-seq shows the efficiency of protection ( $\beta$-glucosyltransferase-mediated protection efficiency equals to $100 \%)$.

Additional file 3. Figure S2. Loci-specific Bis/TAB-seq for HM450K validation. Genomic regions showing agreement in total methylation and hydroxymethylation levels detected by HM450K Bis/OxBis and loci-specific Bis/TAB-seq, respectively. Red dots depict $5 \mathrm{modC}$ (top) and $5 \mathrm{hmC}$ (bottom) levels of each $\mathrm{CpG}$ site detected by loci-specific Bis/TAB-seq, respectively. Blue dots depict 5 modC (top) and $5 \mathrm{hmC}$ (bottom) levels of HM450K $\mathrm{CpG}$ probes. A Regions with significant hydroxymethylation according to HM450K (SLC12A6_2: chr15: 34,628,635-34,628,921; PEG10: chr7: $94,285,83494,286,118)$. B Regions with no hydroxymethylation according to HM450K. The selected negative regions had a similar range of total methylation values to positive regions and serve as a control to eliminate the differences in $5 \mathrm{hmC}$ detection that could be caused by different levels of total methylation (e.g. efficiency of TET-mediated oxidation).

Additional file 4. Figure S3. Single-molecule total methylation (A) and hydroxymethylation (B) patterns of each region (represented as one vertical blue line) were determined based on the deep loci-specific Bis/TAB-seq, respectively. Methylation patterns were separated into five groups based on the percentage of CpGs per region being methylated and/or hydroxymethylated $(\mathbf{A})$ and hydroxymethylated $(\mathbf{B})$. For each region (vertical blue line), the average total methylation $(\mathbf{A})$ or hydroxymethylation (B) was calculated ( $x$-axis) and the frequency of each pattern was plotted along the $y$-axis (summing up to 100\%). Five different patterns were defined based on the proportion of methylated or hydroxymethylated $\mathrm{CpGs}$ of all CpGs per region (0, 0-10, 10-50, 50-80, 80-100\%).

Additional file 5. Figure S4. WG Bis/OxBis-seq and HM450K Bis/OxBis correlation analysis. A Comparison of $5 \mathrm{modC}$ (Bis, left) and $5 \mathrm{mC}(\mathrm{OxBis}$, right) between WG and HM450K Bis/OxBis across CpG sites interrogated by the HM450K and having at least $10 \times$ coverage on the WG Bis/OxBis. B Boxplots showing the difference in $5 \mathrm{modC}$ (Bis, left), $5 \mathrm{mC}$ (OxBis, middle) and $5 \mathrm{hmC}$ (Bis-OxBis, right) between WG and HM450K Bis/OxBis at different levels of the corresponding modification. Only CpG sites $(n=42,537)$ considered as significantly hydroxymethylated by both approaches are included. C Boxplots showing the relationship between the total methylation levels according to the WG Bis/OxBis ( $x$-axis) and the difference in $5 \mathrm{mC}($ left) and $5 \mathrm{hmC}(r i g h t)$ between approaches. The difference is calculated as HM450K methylation value subtracted from the WG methylation value (y-axis),

Additional file 6. Figure S5. Validation of the hMeDIP-seq approach with the WG Bis/OxBis-seq. A The histogram showing the distribution of the hMeDIP-seq signal normalized to the input (logFC). The colours indicate the cut-offs set to depict $\operatorname{low}(\log F C<1.8$ ), medium ( $\log F C>1.8$ and <2.4) and high (log FC > 2.4) hMeDIP signal enrichment. B The distribution of WG-derived $5 \mathrm{hmC}$ levels at hMeDIP summits expanded \pm 150 bp binned based on their logFC values; and gaps between them. C Screen shots of genomic regions showing specific (above) and nonspecific (below) hMeDIP-seq peaks.

Additional file 7. Figure S6. TAB-Seq amplicon validation of hMeDIPseq in cell line DNA. Screen shots showing hMeDIP-seq peaks. WG Bis/ OxBis data are shown. Grey denotes regions not covered $>10 \times$ by WG Bis/ OxBis. Locus-specific TAB-Seq validation showing mod C ( $5 \mathrm{mC}+5 \mathrm{hmC})$ and $5 \mathrm{hmC}$ across the amplicons with $\% 5 \mathrm{mC}$ and $\% 5 \mathrm{hmC}$ for each $\mathrm{CpG}$ site indicated.

Additional file 8. Table S2. Primers.

\section{Abbreviations}

5hmC: 5-hydroxymethylcytosine; 5mC: 5-methylcytosine; 5 modC: total methylation $(5 \mathrm{mC}+5 \mathrm{hmC})$; TAB-seq: TET-assisted bisulphite sequencing; Bis-seq: bisulphite sequencing; OxBis: oxidative bisulphite;WG: whole genome.

\section{Authors' contributions}

KS, CS and SJC were involved in conception and design. KS, EZ, CMG and PLL were involved in analysis and interpretation of data (e.g. statistical analysis, computational analysis). SSN and KS performed WG Bis/OxBis and hMeDIP. $\mathrm{KS}, \mathrm{CS}, \mathrm{EZ}$ and SJC wrote and reviewed the manuscript. All authors read and approved the final manuscript.

\section{Author details}

${ }^{1}$ Epigenetics Research Laboratory, Genomics and Epigenetics Division, Garvan Institute of Medical Research, 384 Victoria Street, Darlinghurst, Sydney, NSW 2010, Australia. ${ }^{2}$ St Vincent's Clinical School, UNSW Australia, Sydney, NSW 2010, Australia. 


\section{Acknowledgements}

We thank Madhavi Maddugoda for the preparation of figures and reviewing the manuscript. We thank Cambridge Epigenetix (CEGX) for conducting the HM450K Bis and OxBis array experiments.

\section{Competing interests}

The authors declare that they have no competing interests.

\section{Availability of data and materials}

The data generated as part of this study are available at the Gene Expression Omnibus (GEO) under accession GSE94368.

\section{Ethics approval and consent to participate}

Commercially available brain DNA was used for the study.

\section{Funding}

This work was supported by National Health and Medical Research Counci (NHMRC) project Grant (\#1088144); NHMRC Fellowship (S.J.C.) (\#1063559); Cancer Australia project Grant (\#1044458). The contents of the published material are solely the responsibility of the administering institution and individual authors and do not reflect the views of the NHMRC.

Received: 16 January 2017 Accepted: 22 March 2017

Published online: 20 April 2017

\section{References}

1. Bird AP. CpG-rich islands and the function of DNA methylation. Nature. 1986;321:209-13.

2. Li E, Beard C, Jaenisch R. Role for DNA methylation in genomic imprinting. Nature. 1993;366:362-5.

3. Jones PA, Baylin SB. The epigenomics of cancer. Cell. 2007;128:683-92.

4. Shen L, Inoue A, He J, Liu Y, Lu F, Zhang Y. Tet3 and DNA replication mediate demethylation of both the maternal and paternal genomes in mouse zygotes. Cell Stem Cell. 2014;15:459-70.

5. Guo F, Li X, Liang D, Li T, Zhu P, Guo H, Wu X, Wen L, Gu TP, Hu B, et al. Active and passive demethylation of male and female pronuclear DNA in the mammalian zygote. Cell Stem Cell. 2014;15:447-58.

6. Ficz G, Branco MR, Seisenberger S, Santos F, Krueger F, Hore TA, Marques CJ, Andrews S, Reik W. Dynamic regulation of 5-hydroxymethylcytosine in mouse ES cells and during differentiation. Nature. 2011;473:398-402.

7. Koh KP, Yabuuchi A, Rao S, Huang Y, Cunniff K, Nardone J, Laiho A, Tahilian M, Sommer CA, Mostoslavsky G, et al. Tet1 and Tet2 regulate 5-hydroxymethylcytosine production and cell lineage specification in mouse embryonic stem cells. Cell Stem Cell. 2011;8:200-13.

8. Lister R, Mukamel EA, Nery JR, Urich M, Puddifoot CA, Johnson ND, Lucero J, Huang Y, Dwork AJ, Schultz MD, et al. Global epigenomic reconfiguration during mammalian brain development. Science. 2013;341:1237905

9. Wang T, Pan Q, Lin L, Szulwach KE, Song CX, He C, Wu H, Warren ST, Jin P, Duan R, Li X. Genome-wide DNA hydroxymethylation changes are associated with neurodevelopmental genes in the developing human cerebellum. Hum Mol Genet. 2012;21:5500-10

10. Ficz G, Gribben JG. Loss of 5-hydroxymethylcytosine in cancer: cause or consequence? Genomics. 2014;104:352-7.

11. Branco MR, Ficz G, Reik W. Uncovering the role of 5-hydroxymethylcytosine in the epigenome. Nat Rev Genet. 2012;13:7-13.

12. Wossidlo M, Nakamura T, Lepikhov K, Marques CJ, Zakhartchenko V, Boiani M, Arand J, Nakano T, Reik W, Walter J. 5-Hydroxymethylcytosine in the mammalian zygote is linked with epigenetic reprogramming. Nat Commun. 2011;2:241

13. Hahn MA, Qiu R, Wu X, Li AX, Zhang H, Wang J, Jui J, Jin SG, Jiang Y, Pfeifer GP, Lu Q. Dynamics of 5-hydroxymethylcytosine and chromatin marks in Mammalian neurogenesis. Cell Rep. 2013;3:291-300.

14. Inoue A, Zhang Y. Replication-dependent loss of 5-hydroxymethylcytosine in mouse preimplantation embryos. Science. 2011;334:194.

15. Pastor WA, Aravind L, Rao A. TETonic shift: biological roles of TET proteins in DNA demethylation and transcription. Nat Rev Mol Cell Biol. 2013;14:341-56.
16. He YF, Li BZ, Li Z, Liu P, Wang Y, Tang Q, Ding J, Jia Y, Chen Z, Li L, et al. Tet-mediated formation of 5-carboxylcytosine and its excision by TDG in mammalian DNA. Science. 2011;333:1303-7.

17. Shen L, Zhang Y. 5-Hydroxymethylcytosine: generation, fate, and genomic distribution. Curr Opin Cell Biol. 2013;25:289-96.

18. Song CX, Yi C, He C. Mapping recently identified nucleotide variants in the genome and transcriptome. Nat Biotechnol. 2012;30:1107-16.

19. Thomson JP, Hunter JM, Lempiainen H, Muller A, Terranova R, Moggs JG, Meehan RR. Dynamic changes in 5-hydroxymethylation signatures underpin early and late events in drug exposed liver. Nucleic Acids Res. 2013:41:5639-54.

20. Bachman M, Uribe-Lewis S, Yang X, Williams M, Murrell A, Balasubramanian S. 5-Hydroxymethylcytosine is a predominantly stable DNA modification. Nat Chem. 2014;6:1049-55.

21. Tahiliani M, Koh KP, Shen Y, Pastor WA, Bandukwala H, Brudno Y, Agarwal S, lyer LM, Liu DR, Aravind L, Rao A. Conversion of 5-methylcytosine to 5-hydroxymethylcytosine in mammalian DNA by MLL partner TET1. Science. 2009:324:930-5

22. Kriaucionis S, Heintz N. The nuclear DNA base 5-hydroxymethylcytosine is present in Purkinje neurons and the brain. Science. 2009;324:929-30.

23. Ito S, Shen L, Dai Q, Wu SC, Collins LB, Swenberg JA, He C, Zhang Y. Tet proteins can convert 5-methylcytosine to 5-formylcytosine and 5-carboxylcytosine. Science. 2011;333:1300-3.

24. Thomson JP, Hunter JM, Nestor CE, Dunican DS, Terranova R, Moggs JG, Meehan RR. Comparative analysis of affinity-based 5-hydroxymethylation enrichment techniques. Nucleic Acids Res. 2013:41:e206.

25. Globisch D, Munzel M, Muller M, Michalakis S, Wagner M, Koch S, Bruckl T, Biel M, Carell T. Tissue distribution of 5-hydroxymethylcytosine and search for active demethylation intermediates. PLoS ONE. 2010:5(12):e15367.

26. Song CX, Szulwach KE, Fu Y, Dai Q, Yi C, Li X, Li Y, Chen CH, Zhang W, Jian $X$, et al. Selective chemical labeling reveals the genome-wide distribution of 5-hydroxymethylcytosine. Nat Biotechnol. 2011;29:68-72.

27. Williams K, Christensen J, Pedersen MT, Johansen JV, Cloos PA, Rappsilber J, Helin K. TET1 and hydroxymethylcytosine in transcription and DNA methylation fidelity. Nature. 2011;473:343-8.

28. Wu H, D'Alessio AC, Ito S, Wang Z, Cui K, Zhao K, Sun YE, Zhang Y. Genome-wide analysis of 5-hydroxymethylcytosine distribution reveals its dual function in transcriptional regulation in mouse embryonic stem cells. Genes Dev. 2011;25:679-84.

29. Booth MJ, Branco MR, Ficz G, Oxley D, Krueger F, Reik W, Balasubramanian S. Quantitative sequencing of 5-methylcytosine and 5-hydroxymethylcytosine at single-base resolution. Science. 2012:336:934-7.

30. Yu M, Hon GC, Szulwach KE, Song CX, Zhang L, Kim A, Li X, Dai Q, Shen Y, Park $B$, et al. Base-resolution analysis of 5-hydroxymethylcytosine in the mammalian genome. Cell. 2012;149:1368-80.

31. Field SF, Beraldi D, Bachman M, Stewart SK, Beck S, Balasubramanian S. Accurate measurement of 5-methylcytosine and 5-hydroxymethylcytosine in human cerebellum DNA by oxidative bisulfite on an array (OxBS array). PLOS ONE. 2015:10:e0118202.

32. Nazor KL, Boland MJ, Bibikova M, Klotzle B, Yu M, Glenn-Pratola VL, Schell JP, Coleman RL, Cabral-da-Silva MC, Schmidt U, et al. Application of a low cost array-based technique-TAB-Array_-for quantifying and mapping both $5 \mathrm{mC}$ and $5 \mathrm{hmC}$ at single base resolution in human pluripotent stem cells. Genomics. 2014;104:358-67.

33. Ruzov A, Tsenkina Y, Serio A, Dudnakova T, Fletcher J, Bai Y, Chebotareva T, Pells S, Hannoun Z, Sullivan G, et al. Lineage-specific distribution of high levels of genomic 5-hydroxymethylcytosine in mammalian development. Cell Res. 2011;21:1332-42.

34. Ernst J, Kellis M. ChromHMM: automating chromatin-state discovery and characterization. Nat Methods. 2012:9:215-6.

35. Ernst J, Kheradpour P, Mikkelsen TS, Shoresh N, Ward LD, Epstein CB, Zhang $X$, Wang L, Issner R, Coyne M, et al. Mapping and analysis of chromatin state dynamics in nine human cell types. Nature. 2011;473:43-9.

36. Bibikova M, Le J, Barnes B, Saedinia-Melnyk S, Zhou L, Shen R, Gunderson $\mathrm{KL}$. Genome-wide DNA methylation profiling using Infinium(R) assay. Epigenomics. 2009;1:177-200.

37. Bibikova M, Barnes B, Tsan C, Ho V, Klotzle B, Le JM, Delano D, Zhang L, Schroth GP, Gunderson KL, et al. High density DNA methylation array with single CpG site resolution. Genomics. 2011;98:288-95.

38. Stirzaker C, Taberlay PC, Statham AL, Clark SJ. Mining cancer methylomes: prospects and challenges. Trends Genet. 2014;30:75-84. 
39. Pidsley R, Zotenko E, Peters TJ, Lawrence MG, Risbridger GP, Molloy P, Van Djik S, Muhlhausler B, Stirzaker C, Clark SJ. Critical evaluation of the Illumina MethylationEPIC BeadChip microarray for whole-genome DNA methylation profiling. Genome Biol. 2016;17:208.

40. Clark SJ, Harrison J, Paul CL, Frommer M. High sensitivity mapping of methylated cytosines. Nucleic Acids Res. 1994;22:2990-7.

41. Huang Y, Pastor WA, Shen Y, Tahiliani M, Liu DR, Rao A. The behaviour of 5-hydroxymethylcytosine in bisulfite sequencing. PLOS ONE. 2010;5:e8888.

42. Jin SG, Kadam S, Pfeifer GP. Examination of the specificity of DNA methylation profiling techniques towards 5-methylcytosine and 5-hydroxymethylcytosine. Nucleic Acids Res. 2010;38:e125.

43. Yu M, Hon GC, Szulwach KE, Song CX, Jin P, Ren B, He C. Tet-assisted bisulfite sequencing of 5-hydroxymethylcytosine. Nat Protoc. 2012;7:2159-70.

44. Nestor CE, Ottaviano R, Reinhardt D, Cruickshanks HA, Mjoseng HK, McPherson RC, Lentini A, Thomson JP, Dunican DS, Pennings S, et al. Rapid reprogramming of epigenetic and transcriptional profiles in mammalian culture systems. Genome Biol. 2015;16:11.

45. Ziller MJ, Hansen KD, Meissner A, Aryee MJ. Coverage recommendations for methylation analysis by whole-genome bisulfite sequencing. Nat Methods. 2015:12:230-2.

46. Han D, Lu XY, Shih AH, Nie J, You QC, Xu MM, Melnick AM, Levine RL, He C. A highly sensitive and robust method for genome-wide $5 \mathrm{hmC}$ profiling of rare cell populations. Mol Cell. 2016;63:711-9.
47. Song JZ, Stirzaker C, Harrison J, Melki JR, Clark SJ. Hypermethylation trigger of the glutathione-S-transferase gene (GSTP1) in prostate cancer cells. Oncogene. 2002;21:1048-61.

48. Consortium B. Quantitative comparison of DNA methylation assays for biomarker development and clinical applications. Nat Biotechnol. 2016;34:726-37.

49. Aryee MJ, Jaffe AE, Corrada-Bravo H, Ladd-Acosta C, Feinberg AP, Hansen KD, Irizarry RA. Minfi: a flexible and comprehensive Bioconductor package for the analysis of Infinium DNA methylation microarrays. Bioinformatics. 2014;30:1363-9.

50. Ritchie ME, Phipson B, Wu D, Hu Y, Law CW, Shi W, Smyth GK. limma powers differential expression analyses for RNA-sequencing and microarray studies. Nucleic Acids Res. 2015;43:e47.

51. Langmead B, Trapnell C, Pop M, Salzberg SL. Ultrafast and memoryefficient alignment of short DNA sequences to the human genome. Genome Biol. 2009;10:R25.

52. Robinson MD, McCarthy DJ, Smyth GK. edgeR: a Bioconductor package for differential expression analysis of digital gene expression data. Bioinformatics. 2010;26:139-40.

53. Zhang Y, Liu T, Meyer CA, Eeckhoute J, Johnson DS, Bernstein BE, Nusbaum C, Myers RM, Brown M, Li W, Liu XS. Model-based analysis of ChIP-Seq (MACS). Genome Biol. 2008;9:R137.

\section{Submit your next manuscript to BioMed Central and we will help you at every step:}

- We accept pre-submission inquiries

- Our selector tool helps you to find the most relevant journal

- We provide round the clock customer support

- Convenient online submission

- Thorough peer review

- Inclusion in PubMed and all major indexing services

- Maximum visibility for your research

Submit your manuscript at www.biomedcentral.com/submit 